\title{
A scaling analysis for turbulent shock-wave/boundary-layer interactions
}

\author{
L. J. Souverein ${ }^{1}$, P. G. Bakker ${ }^{2}$ and P. Dupont ${ }^{3, \dagger}$ \\ ${ }^{1}$ Astrium GmbH Space Transportation, Propulsion \& Equipment - Advanced Programmes, Engineering \& \\ Technology, 81663 Munich, Germany \\ ${ }^{2}$ Faculty of Aerospace Engineering, Delft University of Technology, Kluyverweg 1, \\ 2629 HS, Delft, The Netherlands \\ ${ }^{3}$ Institut Universitaire des Systèmes Thermiques Industriels, Aix-Marseille Université and UMR CNRS \\ 7343, Marseille 13013, France
}

(Received 25 January 2012; revised 20 July 2012; accepted 8 October 2012)

A model based on mass conservation properties is developed for shock-wave/boundarylayer interactions (SWBLIs), aimed at reconciling the observed great diversity in flow organization documented in the literature, induced by variations in interaction geometry and aerodynamic conditions. It is the basis for a scaling approach for the interaction length that is valid independent of the geometry of the flow (considering compression corners and incident-reflecting shock interactions). As part of the analysis, a scaling argument is proposed for the imposed pressure jump that depends principally on the free-stream Mach number and the flow deflection angle. Its interpretation as a separation criterion leads to a successful classification of the separation states for turbulent SWBLIs (attached, incipient or separated). In addition, the dependence of the interaction length on the Reynolds number and the Mach numbers is accounted for. A large compilation of available data provides support for the validity of the model. Some general properties on the state of the flow are derived, independent of the geometry of the flow and for a wide range of Mach numbers and Reynolds numbers.

Key words: compressible boundary layers, compressible flows, shock waves

\section{Introduction}

Shock-wave/boundary-layer interactions (SWBLIs) have been widely studied in the past few decades: see for example the review papers of Délery \& Marvin (1986), Viswanath (1988), Dolling (2001), Smits \& Dussauge (2006), Clemens \& Narayanaswamy (2009) and Délery \& Dussauge (2009). The most commonly considered interactions concern those with a turbulent boundary layer, although laminar or transitional interactions have also been investigated in the literature. Cases under consideration cover a large range of geometric configurations, including normal shock interactions (Atkin \& Squire 1992; Bruce \& Babinsky 2008; Bur et al. 2008), blunt fin interactions (Brusniak \& Dolling 1994; Ünalmis \& Dolling 1996; Bueno 2006), over-expanded nozzles (Frey \& Hagemann 1998, 2000; Bourgoing \& Reijasse 2005), compression ramp interactions (Thomke \& Roshko 1969; Spaid \&

$\dagger$ Email address for correspondence: pierre.dupont@ polytech.univ-mrs.fr 
Frishett 1972; Settles, Fitzpatrick \& Bogdonoff 1979; Debiève 1983; Ardonceau 1984; Dolling \& Or 1985; Smits \& Muck 1987; Kuntz, Amatucci \& Addy 1987; Selig et al. 1989; Erengil \& Dolling 1991a,b; Thomas, Putman \& Chu 1994; Beresh, Clemens \& Dolling 2002; Hou 2003; Ganapathisubramani, Clemens \& Dolling 2007a; Wu \& Martin 2008; Ringuette, Wu \& Martin 2008) and incident-reflecting shock interactions (Green 1970; Dupont, Haddad \& Debiève 2006; Pirozzoli \& Grasso 2006; Touber \& Sandham 2008; Piponniau et al. 2009; Humble et al. 2009a; Souverein et al. 2009; Polivanov, Sidorenko \& Maslov 2009; Garnier 2009; Touber \& Sandham 2009a; Pirozzoli et al. 2009; Souverein et al. 2010; Souverein 2010). The list is evidently not exhaustive. In addition, the considered flow conditions cover a large range of Mach numbers (from transonic to hypersonic values) and Reynolds numbers (spanning at least two orders of magnitude depending on the experimental facilities).

Despite these large variations in aerodynamic and geometric parameters, several general properties have been highlighted for this family of flows. The qualitative mean organization of the flow is currently quite well understood: see Délery \& Marvin (1986). Good quantitative agreement has been obtained in the particular case where the shock strength is large enough for the boundary layer to separate. In those cases, the free interaction theory proposed by Chapman, Kuehn \& Larson (1957), suggests that the separation shock properties become independent of the original cause of the separation (i.e. the flow deflection angle). Therefore, notwithstanding the difference in flow geometry, the wall pressure distributions for sufficiently separated shock reflections and compression ramps are nearly coincident (see Délery \& Marvin 1986). However, no satisfactory description has yet been found that relates the extent of the interaction to the combined effect of variations in the Mach number, the Reynolds number, the state of the incoming boundary layer (including non-adiabatic wall conditions and upstream control) and the imposed adverse pressure gradient. Results are even worse when the geometry is also included as a parameter.

More recently, evidence of low-frequency unsteadiness of the separation shock has been reported (Dolling \& Murphy 1983; Dolling \& Brusniak 1989; Erengil \& Dolling 1991b; Thomas et al. 1994). These frequencies are about two orders of magnitude below the energetic scales of the upstream boundary layer and their origin has been a subject of study for nearly two decades.

Although the precise sources of the separation shock motions are still under debate, it has been shown recently that in separated cases, the low-frequency unsteadiness of the separation shock is also fairly independent of the particular geometry of the flow (Dussauge, Dupont \& Debiève 2006; Dupont et al. 2006; Wu \& Martin 2008; Touber \& Sandham 2009b). For example, a dimensionless frequency of the separation shock oscillations can be defined with a nearly constant value, for any shock-induced separation considered (see Erengil \& Dolling 1991b; Dussauge et al. 2006). This dimensionless frequency, or Strouhal number, is defined by

$$
S_{L}=\frac{f L}{U_{1}},
$$

where $f$ is the characteristic shock motion frequency, $L$ is the characteristic length of the interaction, and $U_{1}$ is the velocity downstream of the separated shock. The Strouhal number $S_{L}$, originally proposed for a Mach 3 compression ramp case (Erengil \& Dolling 1991b), is similar to its counterpart for incompressible separated flows (Kiya \& Sasaki 1983; Cherry, Hillier \& Latour 1984). Typical values of 0.12 are obtained in subsonic separated flows, compared to the nearly constant value of $\sim 0.03$ for supersonic cases at a Mach number larger than 2. 

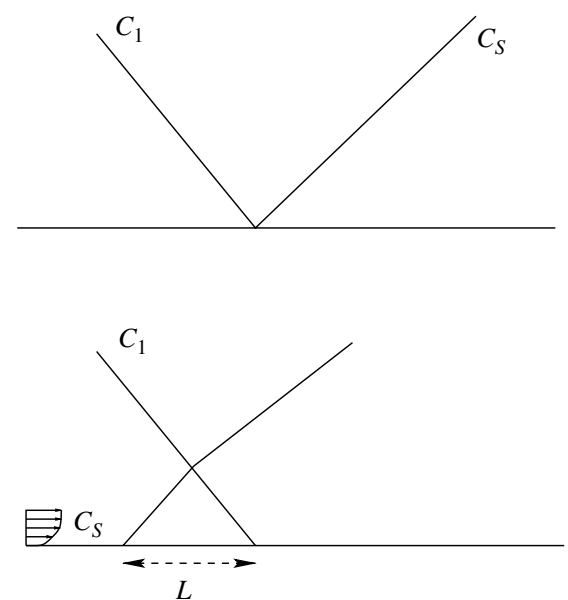
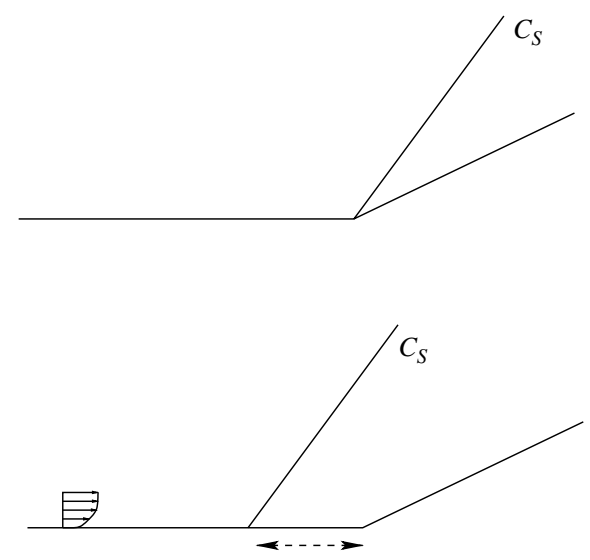

$L$

FIGURE 1. Definition of the interaction length $L$ for reflection and compression ramp cases.

The characteristic length $L$ used in the Strouhal number represents the effects of the presence of the boundary layer in comparison to a purely inviscid flow (see the scheme in figure 1). It is defined as the observed upstream shift of the shock wave $C_{S}$ due to the thickening of the boundary layer, subject to the imposed pressure jump (or equivalently the angle of deviation of the flow). When the boundary layer is sufficiently decelerated to separate, the shock wave $C_{S}$ is called the separation shock. For simplicity, we will keep this nomenclature for this shock wave, whatever the separation state of the flow. Hence, in the case of reflecting waves, $L$ is defined as the distance between the foot of the separation shock and the the extrapolated wall impact point of the incident shock. Similarly, in cases of compression corners and blunt fins, for example, $L$ is defined as the distance between the foot of the separation shock and the corner, respectively the obstacle. It has to be noted that the derivation of the location of the foot of the separation shock can differ depending on the authors and the published data. It has sometimes been derived from Schlieren visualizations, from particle image velocimetry (PIV) data, from the mean wall pressure distribution, or it is associated with the peak in the wall pressure fluctuations, $p_{w}^{\prime}$. This, of course, will introduce some discrepancies in the estimation of $L$. It will, however, be shown that these are significantly less than the observed aerodynamic and geometric effects. In cases with large separation, $L$ can be considered as a rough estimate of the length of separation $L_{\text {sep }}$, which is poorly documented in the literature.

Several attempts have been made to relate the low-frequency shock motions to upstream or downstream unsteadiness. In high-Reynolds compression corner or shock reflection configurations $\left(R e_{\theta} \simeq 5 \times 10^{4}\right.$, where $R e_{\theta}$ is the Reynolds number of the upstream boundary layer based on the momentum thickness), several experimental studies have suggested relating the separation shock motions to unsteady very large structures developed in the upstream boundary layer (Ganapathisubramani et al. 2007a; Humble et al. 2009a). In contrast, experimental and numerical studies performed at low Reynolds numbers $\left(R e_{\theta} \simeq 5 \times 10^{3}\right)$ indicate that the low-frequency shock motions are related to the dynamics of the separated bubble which forms downstream of the separation shock (Dupont et al. 2006; Touber \& Sandham 2008; Wu \& Martin 2008; Piponniau et al. 2009). 
Recently a simple model has been proposed relating the value of the Strouhal number to the entrainment properties of the mixing layer that develops over this separated region (Piponniau et al. 2009). This model suggests a major influence of the compressibility effects on the decrease of this Strouhal number from its subsonic value $(\approx 0.12)$ to its asymptotic value $(\approx 0.03)$ for free-stream Mach numbers larger than 2. Taking into account these compressibility effects and the classical effects of the velocity and density ratios across the mixing layer, this model successfully describes the behaviour of the Strouhal number for a large number of separated flows, ranging from subsonic to hypersonic flows, and for a wide range of Reynolds numbers. Other works propose considering the whole interaction as a dynamic system with its own transfer function. Initially suggested in Plotkin (1975), this approach has been recently reconsidered by Touber \& Sandham (2011) in the case of a Mach 2.3 shock reflection, where the interaction is proposed to act as a low-pass filter for the upstream perturbations. This analysis, based on the similarity properties of the flow inside the first part of the interaction, estimated with success the characteristic time scale of shock unsteadiness in the IUSTI (Institut Universitaire des Systèmes Thermiques Industriels) $8^{\circ}$ interaction. These two models differ mainly in the nature of the low-frequency unsteadiness. Touber and Sandham's model suggests a broad-band mechanism without a particular time scale, limited by the equivalent cut-off frequency of the system. In contrast, the model of Piponniau et al. suggests the existence of a characteristic frequency, eventually with some time fluctuations, due to the turbulence and/or some harmonics in the entrainment time scales. The two types of unsteadiness could even coexist, as suggested in Touber \& Sandham (2011).

In these two last models, the basic driving mechanism for the unsteadiness is derived from the analysis of the mixing layer observed downstream of the separation point in all flows with shock-induced mean separation. The model of Piponniau et al. considers the entrainment influence of the mixing layer whereas the model of Touber and Sandham is based on its similarity properties. Nevertheless, the mechanisms driving the unsteadiness in the case without mean separation are not intuitively evident. Recent experiments on incipiently separated shock reflection interactions were carried out in the low-Reynolds supersonic wind tunnel of IUSTI and in the high-Reynolds wind tunnel of TU Delft (Souverein et al. 2009, 2010) to shed light on this issue. A large Reynolds number range has been considered $\left(4000<R e_{\theta}<\right.$ $50000)$. In these cases, no mean separation is obtained, but instantaneous reverse flow regions can be observed. These experiments show that incipient cases display large similarities and that the Reynolds-number effects on the global properties of the interaction are rather limited. For example, a large resemblance was found in the turbulent fields. Moreover, in both incipient cases, evidence of the development of a mixing layer region downstream of the reflected shock has been given, with a spatial development that resembles the separated cases, with indications of the existence of low-frequency shock motions. However, notwithstanding the presence of the mixing layer, unsteadiness in the upstream boundary layer was also shown to play a role in flows without mean separation.

Despite the successful scaling of the low-frequency unsteadiness based on the characteristic length $L$, no satisfactory scaling of $L$ itself has been derived so far for turbulent SWBLIs. For example, despite similar geometry, no direct comparison of the length of the interactions could be made for the two above-mentioned reflection cases with incipient separation. It will be shown that this is even worse when different geometries are considered. 


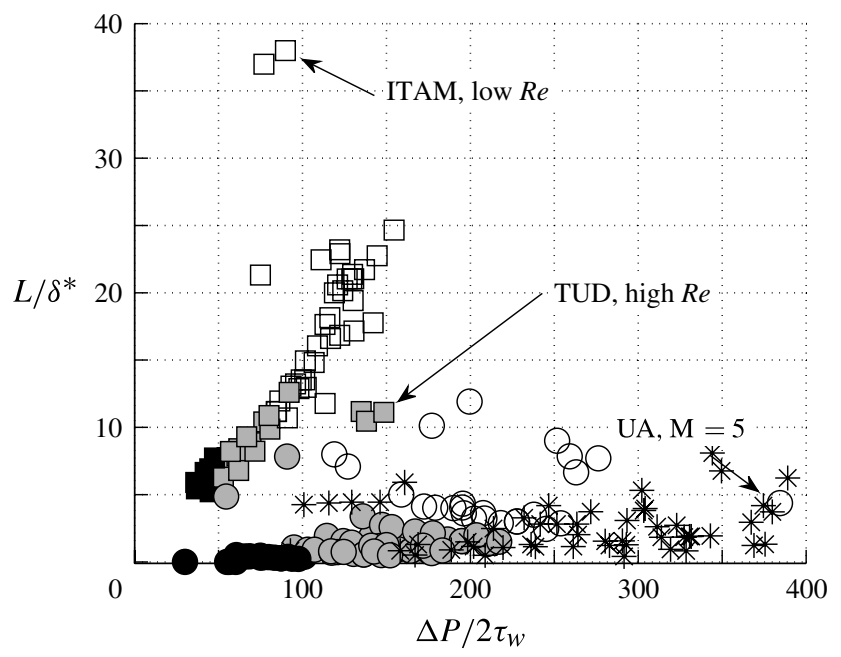

FIGURE 2. Scaling, $L / \delta^{*}$ versus $\Delta P / 2 \tau_{w}$ : $\square$, incident shock reflections; $\bigcirc$, compression ramps. See references in tables 1 and 3 for a complete list of the data sets included. The colours represent the separation state: black, attached; grey, incipient; white, separated.

Given the discussion above, it seems desirable to obtain a better description of the characteristic length $L$, both for the quantitative comparison of different interaction geometries under varying flow conditions, and due to its role as a scaling parameter for the low-frequency unsteadiness. In this article, we propose to compare the characteristic length scales of SWBLIs for various flow conditions and separation states. Of particular interest is the dependence of the characteristic length on the upstream boundary layer and the imposed pressure jump. A simple analysis is made based on a global mass budget along the interaction to derive the parametric dependence for this sort of flows, whatever the geometric conditions (compression corner, incident shock wave ...) and Mach and Reynolds number. The model will be presented in $\S 2$, and then compared with experimental and numerical results obtained for a wide range of Mach and Reynolds numbers, for compression corners and incident shock reflections. The parametric dependence will be discussed and verified against the data available in the literature in $\S 3$. Finally, the possibility of using the new length scaling for the analysis of turbulence fields will be considered in two oblique-shock-wave/turbulent-boundary-layer interactions. The first interaction concerns the low-Reynolds-number Mach 2.3 flow case of IUSTI, and the second one the high-Reynolds-number Mach 1.7 flow case of TU Delft.

\section{Scaling arguments for SWBLIs}

\subsection{Length of interactions in various SWBLIS}

SWBLIs present large qualitative similarities for any aerodynamic and geometric parameters. Nevertheless, despite their great resemblance and the successful scaling of the unsteadiness time scales based on the Strouhal number, it remains quite difficult to compare the length scales of these interactions. Several scaling approaches have been attempted for the interaction length $L$ and the shock intensity $\Delta P$. An example is given in figure 2. It proposes that $L / \delta^{*} \sim \Delta P / \tau_{w}$, where $\delta^{*}$ is the displacement thickness of the upstream boundary layer and $\tau_{w}$ is the wall shear stress, a reasoning 


\begin{tabular}{|c|c|c|c|c|}
\hline Institute & Literature & $R e_{\theta}$ & $M_{e}$ & Symb \\
\hline \multicolumn{5}{|c|}{ Incident shock reflections } \\
\hline IUSTI $^{\mathrm{a}}$ & $\begin{array}{l}\text { Laurent (1996), } \\
\text { Dupont et al. (2006), } \\
\text { Piponniau et al. (2009), } \\
\text { Piponniau (2009), } \\
\text { Souverein et al. (2010), } \\
\text { Souverein \& Debiève (2010), } \\
\text { Souverein (2010) }\end{array}$ & $5.0 \times 10^{3}$ & 2.3 & $\nabla$ \\
\hline \multicolumn{5}{|c|}{ Compression ramps } \\
\hline $\mathrm{UP}^{\mathrm{b}, 1}$ & $\begin{array}{l}\text { Settles et al. (1976), } \\
\text { Settles et al. (1979) }\end{array}$ & $18-300 \times 10^{3}$ & 2.3 & $\bigcirc$ \\
\hline $\mathrm{UP}^{\mathrm{b}, 2}$ & $\begin{array}{l}\text { Dolling \& Or (1985), } \\
\text { Selig et al. (1989) }\end{array}$ & $70 \times 10^{3}$ & 2.9 & 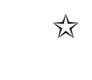 \\
\hline $\mathrm{UND}^{\mathrm{c}}$ & Thomas et al. (1994) & $17.5 \times 10^{3}$ & 1.5 & $\diamond$ \\
\hline $\mathrm{UI}^{\mathrm{d}}$ & Kuntz et al. (1987) & $21 \times 10^{3}$ & 2.9 & $\triangle$ \\
\hline
\end{tabular}

TABLE 1. Interactions considered in the figures to follow: ${ }^{a}$ Institut Universitaire des Systèmes Thermiques Industriels, Marseille; ${ }^{\mathrm{b}}$ University of Princeton; ${ }^{\mathrm{c}}$ University of Notre Dame; ${ }^{\mathrm{d}}$ University of Illinois.

which originates from the free-interaction concept: see Délery \& Marvin (1986). The figure encompasses two flow cases (reflecting shock interactions and compression ramp interactions) for a wide range of flow conditions (Mach number, Reynolds number, flow deflection angle) and flow facilities. Direct numerical simulation (DNS) and large eddy simulation (LES) data are also included. Finally, the state of the interaction, when indicated in the original publication, is used to define the colour of the symbol: black for attached flows, grey for incipient and white for separated ones. Cases where the separation state is unknown, or where uncertainty exists concerning its determination, are indicated with solid symbols (e.g. the asterisks). Some points of interest have been highlighted to illustrate the data range covered by the figure. To be able to compare the ramp flow and reflected shock cases, the pressure rise is defined over the complete interaction. The literature sources and the short names used in this and all the figures to follow are summarized in tables 1 and 3 . Note that the interaction geometry is included as a parameter, since it is believed that the relation between the pressure jump and the interaction length should be properly captured, independent of the particular geometry imposing the pressure jump. This seems to be a reasonable point of view, given the previously recalled observations in the literature (notably the free-interaction concept) that, notwithstanding the difference in flow geometry, the wall pressure distributions nearly coincide, at least in the case of compression ramps and incident shock reflections.

The scaling collapses shock reflection data from the IUSTI S8-facility and data from a similar facility at ONERA taken under close measurement conditions. Both also incorporate the effect of a heated wall, with $T_{w} / T_{a w}=2$, where $T_{w}$ and $T_{a w}$ are respectively the heated wall and the adiabatic wall temperature (see Laurent 1996; Benkemoun \& Salaun 1988). The resulting curve shows a monotonically increasing dependence of $L$ on $\Delta P$. 
The data overview shown in figure 2 is subject to the accuracy of several flow parameters, where the principal uncertainty is expected in the determination of the length scales and the boundary-layer state: the upstream displacement thickness $\delta^{*}$, the wall friction $\tau_{w}$ and the length of interaction $L$ (it is assumed that the freestream Mach number and the flow deflection angle are setting parameters that are known with sufficient accuracy). For example, the University of Princeton highReynolds ramp flow (excluding the data of Settles, Bogdonoff \& Vas 1976) exhibits variations in the specified incoming boundary-layer properties from one reference to the other. As stated by Selig et al. (1989), these variations do not correspond to physically different boundary-layer states, but are due to differences in definitions and determination methods. The scatter in the reported values of $\delta^{*}$ amounts to $\pm 15 \%$. The uncertainty in the determination of the interaction length is reasonably no less than $5 \%$. In addition, the interaction length for attached flows is non-zero, even though the separation length vanishes: in compression ramp cases, where $L$ is often derived from the separation point, an offset near 0 will hence appear. Given these uncertainties and the wide range of measurement techniques, it should therefore be expected that any attempt to collapse data onto a single curve will be faced with an experimental disparity that could amount to as much as $\pm 20 \%$.

Nevertheless, considering interactions with the same separation state (attached/separated), the values of $L / \delta^{*}$ and $\Delta P / 2 \tau_{w}$ show a disparity of up to $500 \%$. Moreover, we remark that there is a general Reynolds number trend in the figures from top left (low-Reynolds cases) to bottom right (high-Reynolds cases). In addition, there seems to be a Mach-number dependence, with the highest Mach-number cases located towards the extreme right. The scaling clearly does not correctly take into account the Reynolds-number effects, the Mach-number effects and the different interaction geometries (incident-reflecting shock and compression ramp). Note that comparable results are obtained when scaling $L$ with the upstream boundary-layer thickness $\delta_{0}$, an approach that was applied with some success in Dupont et al. (2006).

Given the above results we conclude that there exists a relation between the interaction length $L$ and the shock intensity $\Delta P$, but that the scaling with $\delta_{0}$ or $\delta^{*}$ and $\tau_{w}$, respectively, is not satisfactory. Data taken under the same reference conditions do indeed collapse onto a single curve, but the scaling breaks down when the Mach number, Reynolds number or interaction geometry change.

This might indicate that the physical cause of the upstream displacement of the reflected shock is not properly captured, and that the origin of this displacement is not limited to an upstream reference scale. Redefining only one of the axes in figure 2 cannot cause the curves to collapse in a way that makes physical sense: only redefining the scaling for either $\Delta P$ or $L$ may make all data collapse, but in the best case, the most separated compression ramp interactions will collapse with the attached incident-reflecting shock cases. A new scaling must therefore be defined for both $\Delta P$ and $L$ : this is the core of the present work.

As a starting point, we assume that $L$ is some function $F_{1}$ of the reference flow conditions (see (2.1))

$$
L=F_{1}\left(M_{e}, \operatorname{Re}_{\theta}, \varphi, \frac{T_{w}}{T_{a w}}, \text { geometry, } \ldots\right),
$$

where $\varphi$ is the flow deviation (the corner angle for compression ramps and the deviation angle across the incident shock in flow reflection cases).

We now attempt to reformulate the expression in (2.1) in such a way as to obtain a relation between a non-dimensional interaction length $\left(L^{*}\right)$ and a non-dimensional 


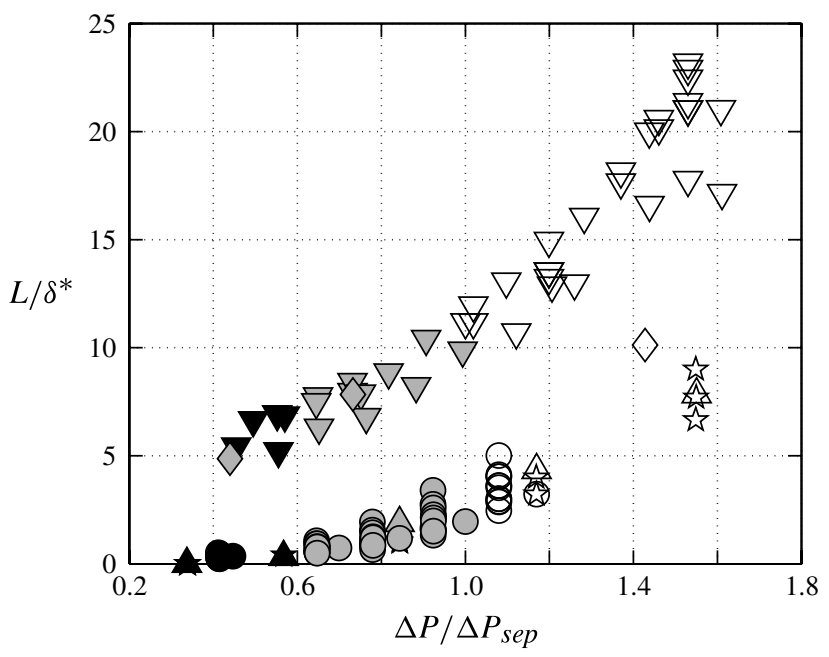

FIGURE 3. Scaling, $L / \delta^{*}$ versus $\Delta P / \Delta P_{\text {sep }}$. Symbols as in table 1 .

interaction strength parameter that represents the tendency of the flow to separate $\left(S^{*}\right)$ : in other words,

$$
L^{*}=F_{2}\left(S^{*}\right),
$$

where $F_{2}$ is a function still to be determined. We propose to define the nondimensional interaction strength parameter $S^{*}$ as

$$
S^{*}=\frac{\Delta P}{\Delta P_{s e p}},
$$

where $\Delta P_{\text {sep }}$ is the shock intensity needed to make the boundary layer separate. It was shown in the previous section that an evaluation of the separation criterion based on the viscous scales of the boundary layer failed to classify the different interactions. Consequently, the difficulty is how to define the shock intensity $\Delta P_{\text {sep }}$ for the different interactions presented in the previous section. For the numerical simulations often only a single flow deviation angle is considered, with a focus on separated cases; no information on the onset of flow separation is available. Similarly, in several experimental works, only one flow deviation is considered. Therefore, for the moment, the only experiments that will be taken into account are those where several flow deviations have been considered (covering all cases from attached to separated conditions) and where the separation state has been determined. This enables the direct evaluation of the separation criterion $S^{*}$ defined by (2.3). The result is illustrated in figure 3, with corresponding references given in table 1 .

It is clear that, notwithstanding the fact that the different interactions are now classified according to their separation state, no satisfying collapse has been obtained for the two geometries, shock reflection and compression corner: the reflection case exhibits a non-dimensional length about 2-4 times larger than the compression corner for equivalent separation states. Moreover, apart from the limited (low) Reynoldsnumber range covered by the subset of shock reflection interactions shown in figure 3 , all data has been obtained at a Mach number of 2.3; hence, a certain effectiveness of the data collapse for this geometry should come as no surprise. Things are different 
for the subset of compression corner experiments, which cover a wider range of Mach numbers (from 2 to 5) and Reynolds numbers (from $2 \times 10^{3}$ to $3 \times 10^{5}$ ), although for this geometry most of the experiments were performed at Mach numbers close to 3. Nevertheless, the experiments from UND clearly diverge from the general trend: these experiments involve lower Mach numbers $(M=1.5)$, suggesting that both the geometry and the Mach number have to be involved in the derivation of $L^{*}$.

In the sections below we will therefore attempt to define a more suitable scaling for the interaction length that represents the relation between $L$ and the change in boundary-layer state induced by $\Delta P$ for use as $L^{*}$ in (2.2). This will be done by considering the integral form of the mass and momentum balance for two interaction cases (incident-reflecting shock and compression ramp). The new scaling will then be formalized by verifying whether it causes all data to fall onto a single curve when plotted against $\Delta P / \Delta P_{\text {sep }}$, representing relation (2.2). Finally, the possibility of deriving an efficient separation state criterion will be considered in $\S 2.3$, and the analysis will be extended to the whole set of data, including the numerical results.

\subsection{Interaction length scaling}

Several investigations have been performed concerning the Mach-number and Reynolds-number effects on the interaction length (see for example Thomke \& Roshko 1969; Spaid \& Frishett 1972; Settles et al. 1976), giving evidence of a dependence on the Reynolds number. Intuitively, it would seem that $\delta^{*}$ could play this role. Nevertheless, it is clear from figure 3 that scaling with $\delta^{*}$ alone does not succeed in collapsing the different geometric configurations, nor does it correctly take into account the Reynolds-number and Mach-number effects. We will therefore attempt to define a more suitable scaling that represents the relation between $L$ and the state of the boundary layer for use as $L^{*}$ in $(2.2)$.

\subsubsection{Model definition and assumptions}

To develop this reasoning further, an inviscid model is defined based on the integral form of the conservation laws. The presence of the boundary layer is taken into account through the integral of the upstream and downstream velocity and density profiles (in other words: $\delta^{*}$ and $\theta$ ). Furthermore, it is assumed that at the exit plane, the pressure jump imposed by the shock system $(\Delta P)$ equals the inviscid value, and that the fluid is parallel to the wall. One may visualize this in terms of the inviscid flow field representation mentioned in Délery \& Marvin (1986) (see figure 4), the difference being that a displacement thickness is added at the wall. The physical cause of the interaction length may now be interpreted as a change in displacement thickness between the upstream and downstream states, creating a kind of 'equivalent' step at the wall. In the current model, we attempt to define a scaling that links the interaction length to the change in boundary-layer state induced by the processes within the interaction resulting from the imposed pressure jump. The downstream evolution of the length scales (due to recovery of the boundary-layer and the boundary-layer growth) are therefore by definition not relevant to the formulation of the scaling. The model is based on a quasi-steady-state assumption and only considers the mean flow. Temporal variations are for the moment not included, but could prove an interesting extension of the model.

To make these ideas concrete, a control volume approach is defined, enclosing the interaction region. It is assumed that the flow is two-dimensional and steady in the mean. In the formulation of the inviscid flow model, the viscous terms are inherently 


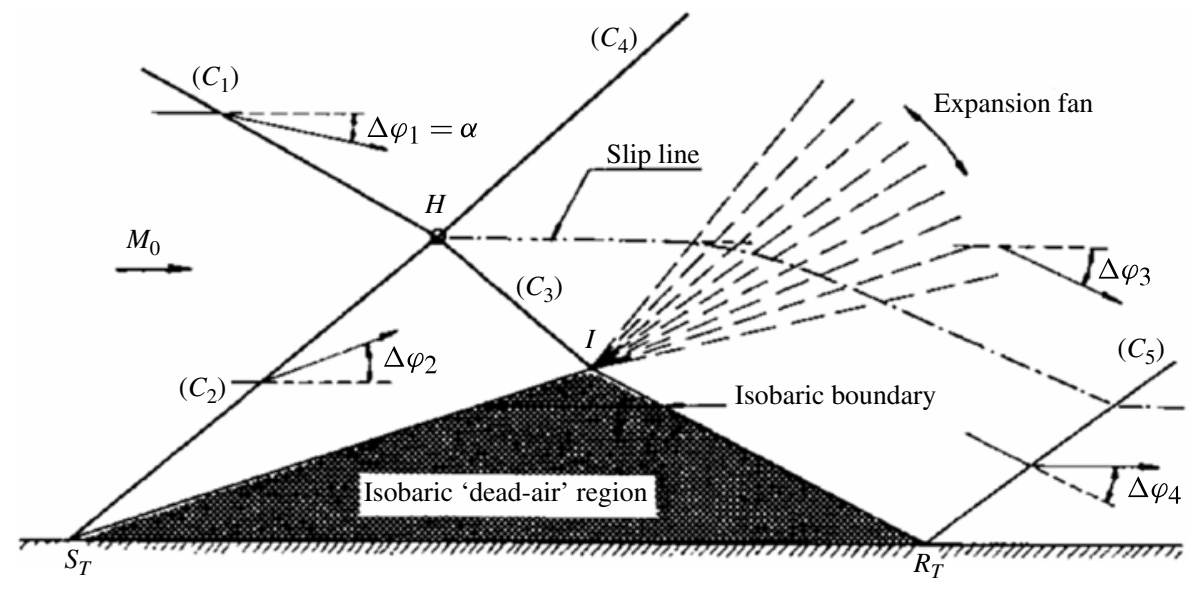

FIGURE 4. Inviscid flow model for the shock-reflection interaction with separation, from Délery \& Marvin (1986).

neglected with respect to the pressure force and the inertial terms in the derivation of a formulation based on momentum conservation.

For the definition of the control volume we do a thought experiment. We imagine we are looking at the interaction from far away, such that $\delta_{0} / L_{c v}, \delta_{0} / H_{c v} \rightarrow 0$, where $L_{c v}$ and $H_{c v}$ are the length and the height of the control volume respectively. In that case, the interaction is reduced to almost a single point and the flow is essentially the same as for the inviscid case. Now let us imagine that we zoom in again, putting ourselves close enough to see the complexities of the interaction, but sufficiently far away that the reflected shock, the expansion fan and the successive recompression waves have all coalesced to form the single shock prescribed by the inviscid flow solution. Putting ourselves in this position, we can model the interaction as a black box that modifies the state of the boundary layer. The flow conditions on all sides of the control volume are prescribed by the free-stream conditions and the inviscid oblique shock relations. The presence of the boundary layer can be taken into account via the definition of the displacement thickness.

In the proposed model, the edge conditions must adhere to the inviscid shock reflection conditions. The pressure gradient imposed by the shock system will lead to a deceleration of the boundary layer and therefore to an increase of the displacement thickness through the interaction as compared with the upstream thickness. Consequently, the only way for the flow to ensure mass and momentum conservation in the presence of the boundary layer is to translate the reflected shock: see figure 5(a) for the reflection case and figure 5(b) for the compression corner. This translation can be cast as an algebraic equation by considering the difference between the inviscid case (the perfect fluid solution without boundary layer and interaction) and the viscous case (with interaction, the viscous effects being modelled as discussed above).

We reiterate the basic assumptions that the shock intensity is the same as in the perfect fluid flow reflection, and that at the outflow plane, the flow conditions outside the boundary layer become uniform and approach the perfect fluid solution. Indeed, in reality one is confronted with deviations from the model assumptions. One may think of the presence of the expansion fan and velocity gradients that curve the 
(a)

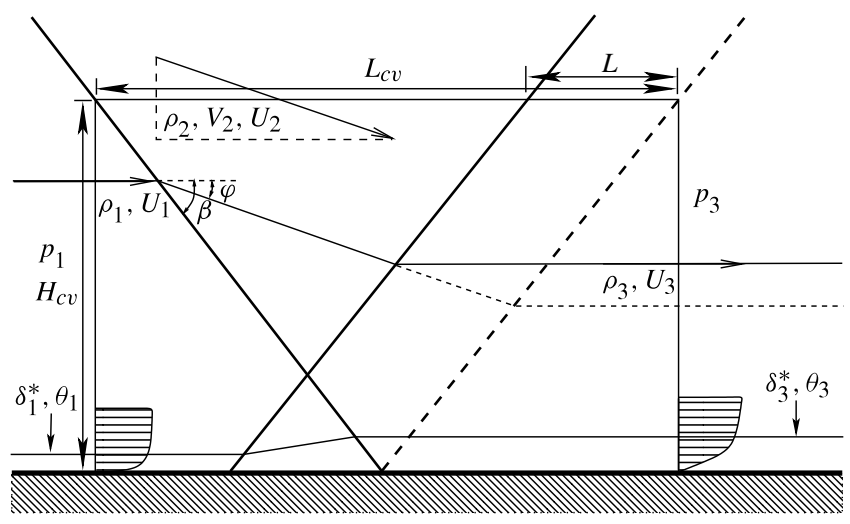

(b)

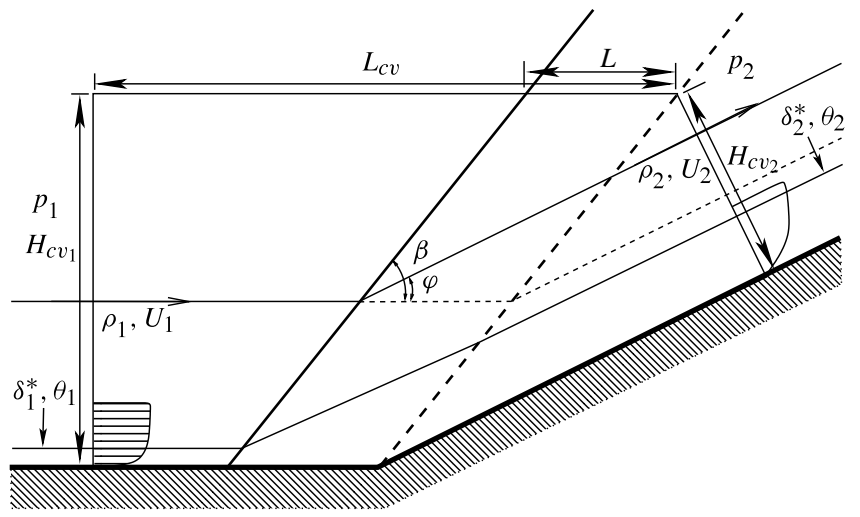

FIGURE 5. Control volume: $(a)$ incident shock reflection case; $(b)$ compression ramp case.

path of the shock within the control volume, inducing for instance a discrepancy between the measured shock position (by means of wall pressure distributions) and the position as defined by the model. In addition, this curving causes entropy gradients, leading to a non-homogeneous velocity and density distribution downstream of the interaction. Although the conservation laws must inherently be satisfied, difficulties are to be expected, particularly when modelling the outflow conditions with an integral boundary-layer length scale in combination with a uniform velocity and density. All these effects will play a role when the model is employed for predictive purposes. However, the current aim is to determine the most dominant mechanisms that govern the interaction length rather than to make precise length estimations. In this context, the deviations are thought to be of only secondary importance. The basic assumptions are therefore believed to be reasonable approximations for the purpose of this work.

We remark that both the mass balance and the momentum balance can be considered individually to derive a physical relation between the upstream and downstream boundary-layer length scales and the interaction length. This has indeed been done, yielding complementary scaling relations because both quantities must be conserved across the interaction. Since the procedure for deriving the two formulations is similar, details will be given here for the mass balance only. The derivation of the formulation based on momentum balance is given in the Appendix. It is also investigated whether a common scaling may be expected for the incident-reflecting shock interactions and the compression ramp flows. 
The two principal geometries - incident shock reflection and compression ramp - are first considered separately. Then we will show that a common formulation can be derived.

\section{Incident shock reflection}

The control volume for the incident-reflecting shock interaction is shown in figure 5(a). Writing the mass flow balance for the inviscid flow case (without interaction, as indicated by the dashed reflecting shock), the following equality is obtained:

$$
\rho_{1} U_{1} H_{c v}+\rho_{2} V_{2} L_{c v}-\rho_{3} U_{3} H_{c v}=0,
$$

where $H_{c v}$ and $L_{c v}$ are the height and length of the control volume respectively. At the same time, the following relation is obtained for the viscous case (with interaction, as indicated by the solid reflecting shock):

$$
\rho_{1} U_{1}\left(H_{c v}-\delta_{1}^{*}\right)+\rho_{2} V_{2}\left(L_{c v}-L\right)-\rho_{3} U_{3}\left(H_{c v}-\delta_{3}^{*}\right)=0 .
$$

Subtracting (2.4) from (2.5), the following algebraic relation for the interaction length is obtained for the shock reflection case:

$$
L=\frac{\rho_{3} U_{3} \delta_{3}^{*}-\rho_{1} U_{1} \delta_{1}^{*}}{\rho_{2} V_{2}} .
$$

The interaction length is hence completely determined via the upstream and downstream boundary-layer displacement thickness, since the densities and velocities are specified by oblique shock-wave theory (depending only on $M_{e}$ and $\varphi$ ). The expression is independent of the height and length of the control volume as long as the interaction is included.

\section{Compression ramp}

The compression ramp case is now considered. The control volume is shown in figure $5(b)$. Writing again the mass-flow balance for the inviscid flow case, the following equality is obtained:

$$
\rho_{1} U_{1} H_{c v_{1}}-\rho_{2} U_{2} H_{c v_{2}}=0 .
$$

At the same time, the following relation is obtained for the case with interaction:

$$
\rho_{1} U_{1}\left(H_{c v_{1}}-\delta_{1}^{*}\right)-\sin (\varphi) \rho_{2} U_{2} L-\rho_{2} U_{2}\left(H_{c v_{2}}-\delta_{2}^{*}\right)=0 .
$$

Subtracting (2.7) from (2.8), the following algebraic relation is obtained for the interaction length for the compression ramp case:

$$
L=\frac{\rho_{2} U_{2} \delta_{2}^{*}-\rho_{1} U_{1} \delta_{1}^{*}}{\sin (\varphi) \rho_{2} U_{2}} .
$$

The interaction length is again completely determined by the upstream and downstream boundary-layer displacement thickness, since the densities and velocities are specified by oblique shock-wave theory (depending only on $M_{e}$ and $\varphi$ ). Again, the expression is independent of the height and length of the control volume as long as the interaction is included.

\subsubsection{Common formulation}

Given the great resemblance between relations (2.6) and (2.9), it is tempting to see whether the two equations can be cast in the same form. To this end, the denominator of each equation is reformulated in terms of the upstream conditions by means of the 
oblique shock-wave relations. The shock angle $\beta$ and the flow deflection angle $\varphi$ are defined to be positive for both interaction cases. Invoking mass conservation across the incident shock for the shock reflection case, the following equality is obtained:

$$
\rho_{1} U_{1} \sin (\beta)=\rho_{2} V_{2} \frac{\sin (\beta-\varphi)}{\sin (\varphi)} .
$$

Similarly, considering mass conservation across the shock for the compression ramp case gives

$$
\rho_{1} U_{1} \sin (\beta)=\rho_{2} U_{2} \sin (\beta-\varphi) .
$$

Rewriting each of the equations above and substituting (using (2.6) for the incident shock interaction and (2.9) for the compression corner) gives the same final equation, which can be cast in the following common form, using the subscripts in and out for the inflow and outflow conditions, respectively:

$$
\frac{L}{\delta_{\text {in }}^{*}}=\frac{\sin (\beta-\varphi)}{\sin (\beta) \sin (\varphi)}\left(\frac{\rho_{\text {out }} U_{\text {out }} \delta_{\text {out }}^{*}}{\rho_{\text {in }} U_{\text {in }} \delta_{\text {in }}^{*}}-1\right) .
$$

The shock angle $\beta$ is an algebraic relation in terms of the flow deflection angle $\varphi$ and the upstream Mach number $M_{e}$. The interaction length is therefore a direct algebraic function of the $M_{e}, \varphi$ and the mass-flow deficit ratio between the incoming boundary layer and the outgoing boundary layer. Defining the mass-flow deficit as $\dot{m}^{*}=\rho U \delta^{*}$, we can hence write

$$
\frac{L}{\delta_{i n}^{*}}=g_{3}\left(M_{e}, \varphi\right)\left(\frac{\dot{m}_{\text {out }}^{*}}{\dot{m}_{\text {in }}^{*}}-1\right) .
$$

Setting $g_{3}\left(M_{e}, \varphi\right)$ as the ratio of sine functions,

$$
g_{3}\left(M_{e}, \varphi\right)=\frac{\sin (\beta-\varphi)}{\sin (\beta) \sin (\varphi)} .
$$

Based on this relation, it can be concluded that the interaction length is a direct result of the mass-flow deficit ratio between the incoming and outgoing boundary layer, and that it can be estimated algebraically when all quantities in the equation are known.

The analysis above based on the mass conservation consideration leads to a physical insight concerning the scaling parameters for the interaction length (the vertical axis of figure 2). Relation (2.13) seems to indicate that the proper scaling of $L$ is indeed with $\delta^{*}$, but that a trigonometrical correction factor is required in the form of function $g_{3}$. In fact, the non-dimensional interaction length represents the change of state between the incoming boundary layer and the outgoing boundary layer. It should be interpreted in terms of the mass-flow deficit ratio. This explains why a simple scaling by a single boundary-layer length scale did not manage to collapse the data. The proper scaling, with $L^{*}$ as the non-dimensional interaction length, would hence be

$$
L^{*}=\frac{L}{\delta_{i n}^{*}} G_{3}\left(M_{e}, \varphi\right)=\frac{\dot{m}_{\text {out }}^{*}}{\dot{m}_{\text {in }}^{*}}-1,
$$

where

$$
G_{3}\left(M_{e}, \varphi\right)=g_{3}^{-1}\left(M_{e}, \varphi\right)=\frac{\sin (\beta) \sin (\varphi)}{\sin (\beta-\varphi)}
$$




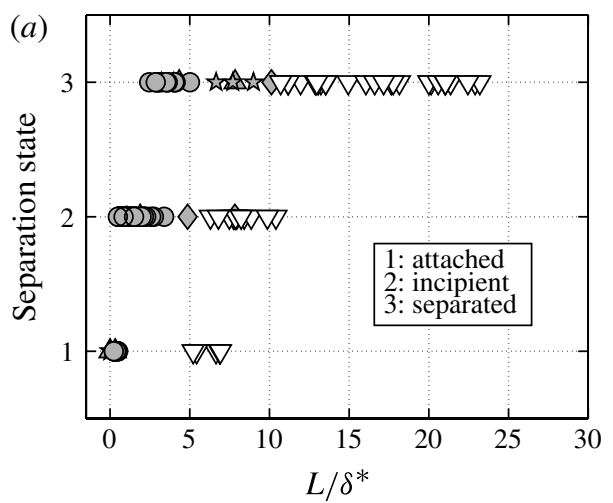

(b)

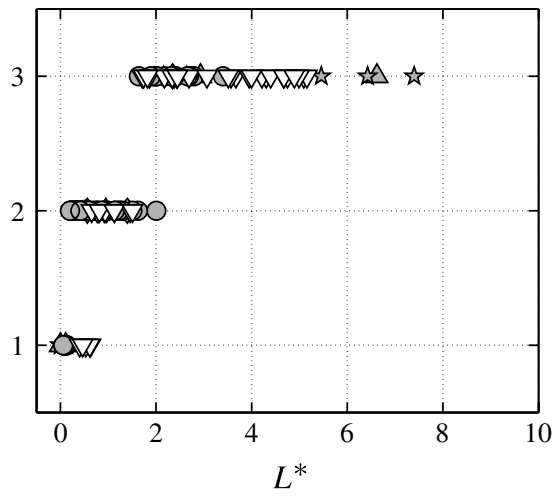

FIGURE 6. Interaction length scaling and documented separation states of the reference interactions: $(a)$ original scaling with $\delta^{*} ;(b)$ new scaling based on mass conservation. Symbols as in table 1. The colours represent the two flow geometries: white, shock reflection; grey, compression ramp.

Hence, $L^{*}$ becomes a function of the mass-flow deficit ratio across the interaction which depends on the adverse pressure gradient imposed on the upstream boundary layer, independently of the geometry and the upstream boundary-layer scales. In order to formalize this relation between $L^{*}$ and the (non-dimensional) pressure gradient $S^{*}, L^{*}$ should therefore classify the different states of the interaction, from attached to separated cases. As a verification, both scaling options, the original $L / \delta^{*}$ and the new scaling $L^{*}$ defined by (2.15), are compared by plotting them against the documented separation state, as was done for the separation criterion in the previous section. The result is shown in figure 6 , with symbols corresponding to table 1 and colours representing the two interaction cases (white, incident-reflecting shock; grey, compression ramp). It is clear that the first choice does not classify the flows. Moreover, the results are largely dependent on the geometry of the flow: shock reflections produce significantly larger interactions whatever the separation state of the flow. In contrast, the new scaling leads to a better classification of the known separation states, and there is a larger consistency between the ramp flow cases and the shock reflection cases, since data corresponding to both geometries are no longer clearly distinct. The attached flow cases take on the value close to zero, as is to be expected from the definition of the scaling: the interaction length becomes small and consequently the mass-flow deficit is only slightly altered. Note that due to the previously mentioned limitations of the compilation concerning the determination of $L$, a value of $L \equiv 0$ is obtained for some of the attached interactions. For compression ramp interactions in particular, the obtained value of $L$ depends strongly on the measurement technique and resolution for (almost) attached flows, and therefore the interaction length vanishes for small flow deflections. Strictly speaking this is not true; however, $L$ will be very small and hence the attached flow cases will indeed approach $L^{*} \downarrow 0$, with a value identical to zero for vanishing shock intensity. The incipient interactions have a non-dimensional interaction length with a value centred around one. The separated interactions take on a value larger than two. These properties are summarized in table 2. Therefore, the proposed scaling seems efficient at comparing the characteristic length scales of the interactions. 


$\begin{array}{ll}\text { Value } & \text { Separation state } \\ L^{*} \downarrow 0 & \text { Attached flow } \\ 1<L^{*}<2 & \text { Incipient separation } \\ L^{*}>2 & \text { Separated flow }\end{array}$

TABLE $2 . L^{*}$ versus the separation states.

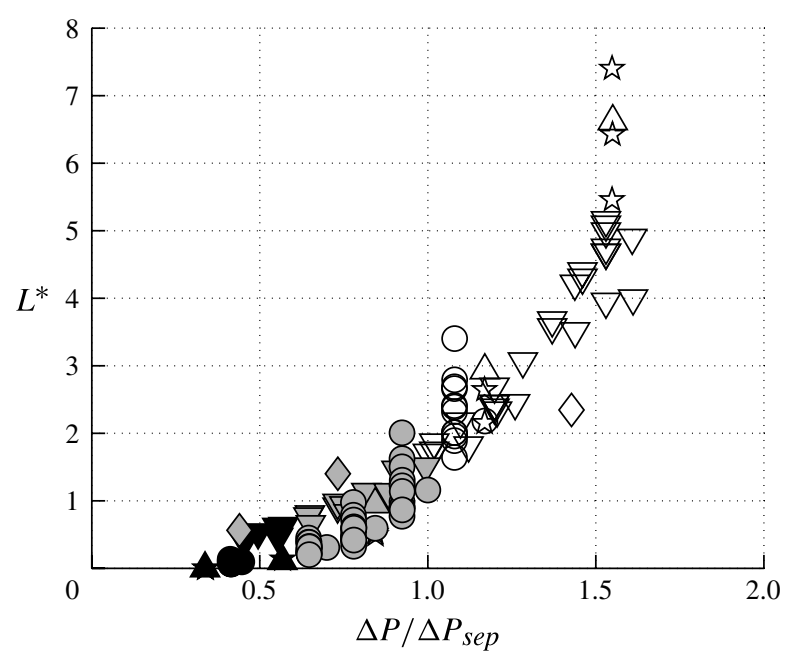

FIGURE 7. New scaling of the interaction length, based on mass balance. Symbols as in table 1. The colours represent the separation state: black, attached; grey, incipient; white, separated.

The new scaling for $L$ is tested in figure 7 . The same symbols are used as in figure 3. The data fall onto a single trend line. In addition, there is a classification of separation states along the curve (attached, incipient, separated), with a progressive increase in the extent of flow separation when moving from left to right along the trend line. The new scaling hence appears to satisfy the desired properties concerning the separation state, while producing a curve that represents the function $F_{2}$, as defined by (2.2).

The only point not collapsing onto the curve is the $\varphi=12^{\circ}$ ramp of Thomas et al. (1994). However, it is difficult to set the onset of separation for this configuration correctly, as the discretization of the flow deviations is quite large: for example, a change of one degree for the onset of separation would place this point in the set of data. Moreover, this ramp configuration is on the very limit between two distinct flow solutions (oblique shock and normal shock), and the post-shock flow has in either case surpassed the sonic limit (the flow behind the shock is hence completely subsonic), raising questions as to the stability of this particular measurement point. It may be expected that the actual flow organization for this interaction is particularly sensitive to small variations in the experimental conditions (upstream and downstream of the interaction), possibly resulting in large deviations from the modelled flow organization.

The effectiveness of the new scaling becomes clear when comparing figure 7 to the scaling from figure 3. Initially, the data points for similar separated states showed a 
disparity of up to $300 \%$, with an even larger difference for the attached cases. The new scaling makes all points follow the same trend, within a scatter of about the same order as the expected measurement uncertainty: see $\S 2.1$.

\subsection{Separation state criteria}

In the previous section we used the separation criterion $S^{*}=\Delta P / \Delta P_{\text {sep }}$ to classify the states of separation of the interactions. Unfortunately, as stated in $\S 2.1$, this criterion requires a priori knowledge of the onset of separation for a given flow case. This makes it impractical for application since one always needs to have information covering a large range of flow deflection angles to be able to derive the scaling for a single SWBLI flow case. Hence, only experiments that provide this information have been included so far, omitting a number of other experimental data sets as well as several recent numerical simulations where $\Delta P_{\text {sep }}$ cannot be determined. To remedy this limitation and to facilitate the application of the scaling to new data points, in this section we attempt to define an effective separation criterion which is valid for the wide range of Mach numbers and Reynolds numbers under consideration, and which is common to the different interaction geometries. This is done on the basis of the experimental data described in the previous section, subsequently investigating its applicability to the other flow cases where the separation onset is unknown.

First considering the tendency of the flow to separate, we note that flow separation is necessarily initiated close to the wall where $\partial P / \partial x \simeq \partial \tau / \partial y$ (in the viscous sublayer). Nonetheless, outside the viscous sublayer we have $\partial P / \partial x \gg \partial \tau / \partial y$. An order of magnitude analysis yields $\Delta P / L \gg \tau_{w} / \delta_{0}$, or equivalently $\Delta P / \tau_{w} \gg L / \delta_{0}$, a condition that is indeed satisfied by the current experiments: see figure 2 . It is therefore justified to consider only the effects of pressure and inertia forces, even though viscous effects exist, albeit confined to a region very close to the wall. From another point of view, $\tau_{w}=\rho_{w} u_{\tau}^{2}$, where $u_{\tau}$ is a measure of the velocity defect in the outer part of the turbulent boundary layer. It is known that $u_{\tau}$ decreases for increasing Reynolds numbers, reducing the velocity defect while increasing $\Delta P / \tau_{w}$ for given Mach number, flow deviation and stagnation temperature. It should be expected that a smaller velocity defect reduces the tendency of the flow to separate. This is in contradiction to the trend indicated by figure 2, which implies that the flow should be more separated for larger $\Delta P / \tau_{w}$. Considering these two elements, it seems that the wall shear stress is not the correct scaling parameter to describe the tendency of the flow to separate, at least not for the Reynolds-number range under consideration, corresponding to fully developed turbulent upstream boundary layers: laminar or transitional interactions are not considered in the current investigation.

Given the above observations, the occurrence of flow separation is expected to be mostly governed by the inertial forces in the incoming flow, at least at sufficiently high Reynolds numbers. To elaborate on this, we attempt to define a scaling for $\Delta P$ based on the Euler equations. Hence, the one-dimensional, steady, inviscid momentum balance will be invoked in its differential form to analyse whether the occurrence of separation can be linked to a ratio between the pressure force and the inertia forces in the incoming flow, with the aim of defining a scaling for $\Delta P$ for use as a separation criterion in (2.2). Of course, this simplified approach has several limitations when applied to the inherently more complicated flow occurring within the interaction. It is done under the hypothesis that it enables us to capture the principal expected driving mechanism for the flow separation state (the balance between pressure and inertia forces) using a simple scaling parameter. Therefore, to evaluate whether this simplified 
approach is indeed in agreement with observations, it will subsequently be verified against the experimental data base.

Under the assumptions stated above, the momentum flux will be related to the pressure gradient as in (2.17):

$$
-\frac{\partial P}{\partial x}=\frac{\partial\left(\rho U^{2}\right)}{\partial x} .
$$

We observe that the adverse pressure gradient $\Delta P$ imposed by the shock system acts approximately over the length of the interaction, decelerating the incoming fluid over the same length. Therefore, the length of interaction $L$ is interpreted as the length scale related to the pressure jump across the interaction. Based on these observations, we propose to relate the pressure jump across the interaction to the momentum decrease as follows:

$$
-\frac{\Delta P}{L} \sim \frac{\Delta\left(\rho U^{2}\right)}{L} .
$$

Recalling the objective of defining a criterion for flow separation, we assume that the flow separates when the incoming streamwise momentum $\rho U^{2}$ vanishes, in other words, when $\Delta\left(\rho U^{2}\right)=\rho U^{2}$. Consequently, at separation, the following relation can be written as

$$
\Delta P_{\text {sep }} \sim \rho_{c} U_{c}^{2},
$$

where $U_{c}$ and $\rho_{c}$ are a reference velocity and density respectively for the incoming momentum flux. Given the previous arguments indicating that the flow is governed by pressure and inertia forces in most of the boundary layer, it is expected that $\rho_{c}$ and $U_{c}$ are of the order of $\rho_{e}$ and $U_{e}$ respectively, where the subscript $e$ is used to refer to the free-stream-based value. Inserting the upstream dynamic pressure $q_{e}=\rho_{e} U_{e}^{2} / 2$, this leads to the separation criterion

$$
S_{e}^{*}=k \frac{\Delta P}{q_{e}},
$$

where $k$ is a constant of order one that will be chosen so as to obtain a value of approximately 1 at the onset of separation.

As stated previously, this analysis constitutes a simplification of the actual flow with the specific aim of defining a separation criterion under the hypothesis of capturing the main driving mechanism. To verify whether this approach is justifiable, we will first compare the separation criterion proposed by (2.20) to the original criterion $S^{*}=\Delta P / \Delta P_{\text {sep }}$ used in the previous section for the subset of the data where the onset of separation was indicated. In figure 8 the results are plotted against the Reynolds number. The results indicate that, despite the large simplifications used to derive the relation (2.20), an efficient estimation of $\Delta P_{\text {sep }}$ can be derived from the upstream dynamic pressure $q_{e}$. First, no remaining influence of the Mach number appears, which means that its influence is accurately taken into account through the dynamic pressure. The ratio is about 3 for the whole set of Reynolds numbers, covering nearly three decades, with a slight decrease over the range of Reynolds numbers from 3 to 2.5, corresponding to $17 \%$. Note that other authors (see Ginoux 1973) have considered with some success the ratio $\Delta P / q_{e}$ to scale the shock intensity. They remark that for turbulent boundary layers at sufficient Reynolds numbers, the occurrence of flow separation is Reynolds-number-independent. The effect of the Reynolds number does indeed seem to depend on its magnitude and consequently the flow regime (laminar, 


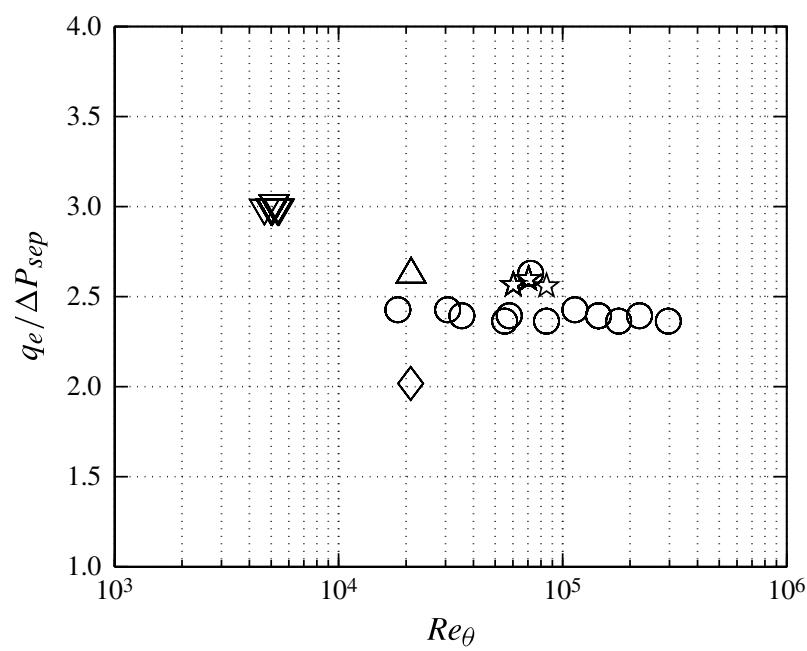

FIGURE 8. Ratio of the dynamic pressure $q_{e}=\rho_{e} U_{e}^{2} / 2$ and the pressure step needed to separate $\Delta P_{\text {sep }}$ versus the Reynolds number of the upstream flow. Symbols as in figure 3.

transitional, turbulent). Moreover, some of the commonly used separation criteria (see Summerfield, Foster \& Swan 1954; Zukoski 1967; Schmucker 1973) are a function of the Mach number and the flow deflection only, implying that the occurrence of separation is Reynolds-number-independent, at least for high Reynolds numbers, in accordance with Ginoux (1973).

Therefore, based on these data we will use the relation (2.20) with $k$ defined by

$$
\begin{aligned}
& k=3.0 \text { if } \operatorname{Re}_{\theta} \leqslant 1 \times 10^{4} \\
& =2.5 \text { if } \operatorname{Re}_{\theta}>1 \times 10^{4} \text {. }
\end{aligned}
$$

Although this definition of $k$ is based on a limited subset of data, including all data from the full compilation shows good agreement with this behaviour, providing further support for the proposed values of $k$ (as will be shown in figure 9). Using the ideal gas law and the definition of the speed of sound, (2.20) can be rewritten as

$$
S_{e}^{*}=\frac{2 k}{\gamma} \frac{\frac{p_{\text {post }}}{p_{\text {pre }}}-1}{M_{e}^{2}},
$$

where $p_{\text {post }} / p_{\text {pre }}$ is the ratio of the pressure before $\left(p_{\text {pre }}\right)$ and after $\left(p_{\text {post }}\right)$ the shock system. For a particular flow organization (for example compression ramp, incidentreflecting shock) and using oblique shock-wave theory (see for example Anderson 1991), it can be written analytically as a function of the free-stream Mach number $M_{e}$, the imposed flow deflection angle $\varphi$ and the specific heat ratio $\gamma$ :

$$
\frac{p_{\text {post }}}{p_{\text {pre }}}-1=f\left(M_{e}, \varphi, \gamma\right) \text {. }
$$

The following final expression is obtained for the separation criterion (assuming a constant specific heat ratio $\gamma$ ):

$$
S_{e}^{*}=\frac{2 k f\left(M_{e}, \varphi, \gamma\right)}{\gamma}=k g_{1}\left(M_{e}, \varphi\right),
$$




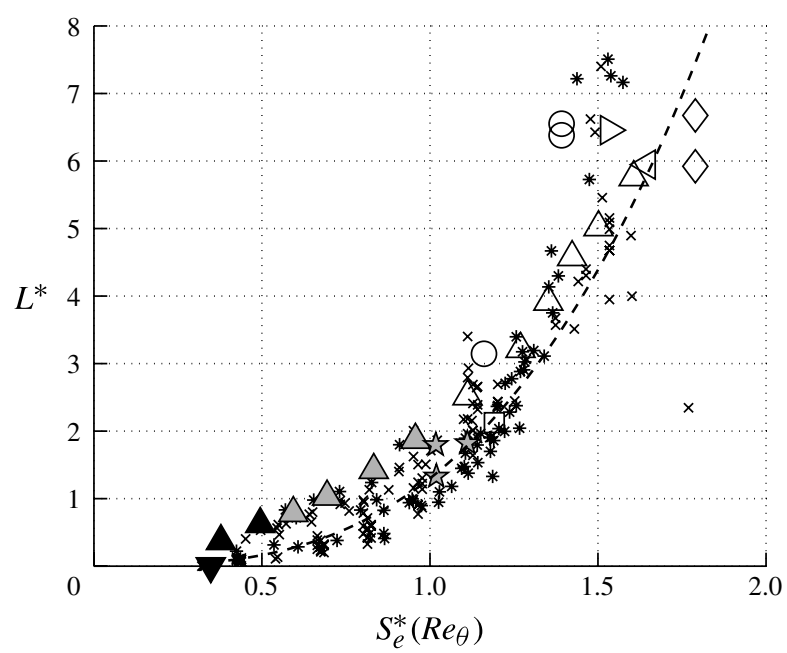

FIGURE 9. New scaling of the interaction length, based on mass balance. The colours represent the separation state: black, attached; grey, incipient; white, separated. Cross symbols, experiments used in figure 3; other symbols given by table 3 . Dashed line, best fit line $\left(a * x^{b}\right.$, with $a=1.3$ and $\left.b=3\right)$.

with $k$ given by the relation (2.21). We reiterate that $g_{1}$ is a function depending on the particular shock system (for example compression ramp, incident-reflecting shock).

In conclusion, we propose that, when turbulent flows are considered, the separation criterion can be represented by an analytic relation depending only on the free-stream Mach number, on the flow deflection angle and on the particular shock system. According to the current formulation, the scaling of the shock intensity and the occurrence of separation for turbulent cases are nearly Reynolds-number-independent, in accordance with the observations from Ginoux (1973). It should therefore be expected that the Reynolds-number effects appear mainly through the scaling of the interaction length through the displacement thickness of the upstream flow (see figure 7). Note that other effects could also influence the onset of separation, such as wall effects (roughness, cooling or heating), as compared to the smooth-wall adiabatic cases considered in the present compilation.

The separation criterion $S_{e}^{*}$ seems to correctly represent the state of the occurrence of flow separation. This makes it a good candidate for $S^{*}$ in (2.2). As it is only based on external quantities, it can be evaluated for the whole set of available experimental and numerical SWBLIs. The results are shown in figure 9. In order to enhance the readability of the picture, the experiments used in figure 7 are consistently represented by a cross symbol, whereas for the new data the symbols are given according to table 3. This new compilation includes NASA-Ames results: see Thomke \& Roshko (1969). These experiments indicate the onset of separation for a wide range of Mach numbers $(2 \leqslant M \leqslant 5)$ at high Reynolds numbers. They were, however, omitted from the previous compilation (see figure 7). The reason for this is that the criterion to qualify the separation state in these experiments was biased towards high angles of deviations (the measurement methods employed in their study are relatively insensitive to small regions of separation), as reported by Settles et al. (1976). As this bias is confirmed by our current compilation, we have not considered them previously in the evaluation of $\Delta P_{\text {sep }}$ and their state of separation is not shown 
总 号

$\triangleright \diamond$

$\triangle \nabla * \square$

4

$\stackrel{i}{*} \quad \stackrel{0}{*}$

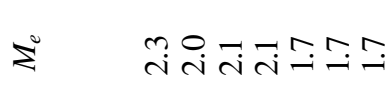

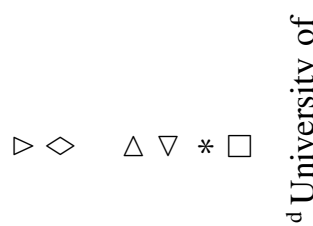

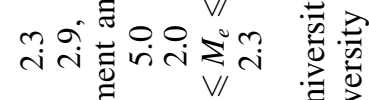

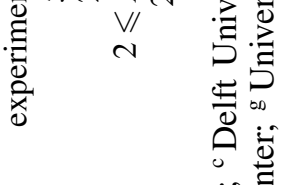

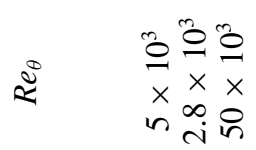

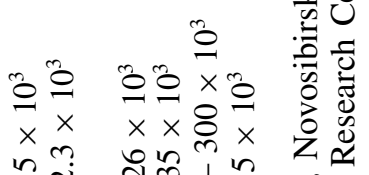

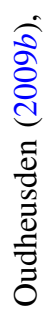

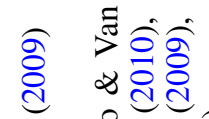

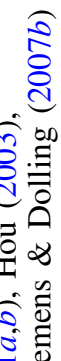

$\stackrel{0}{=}$
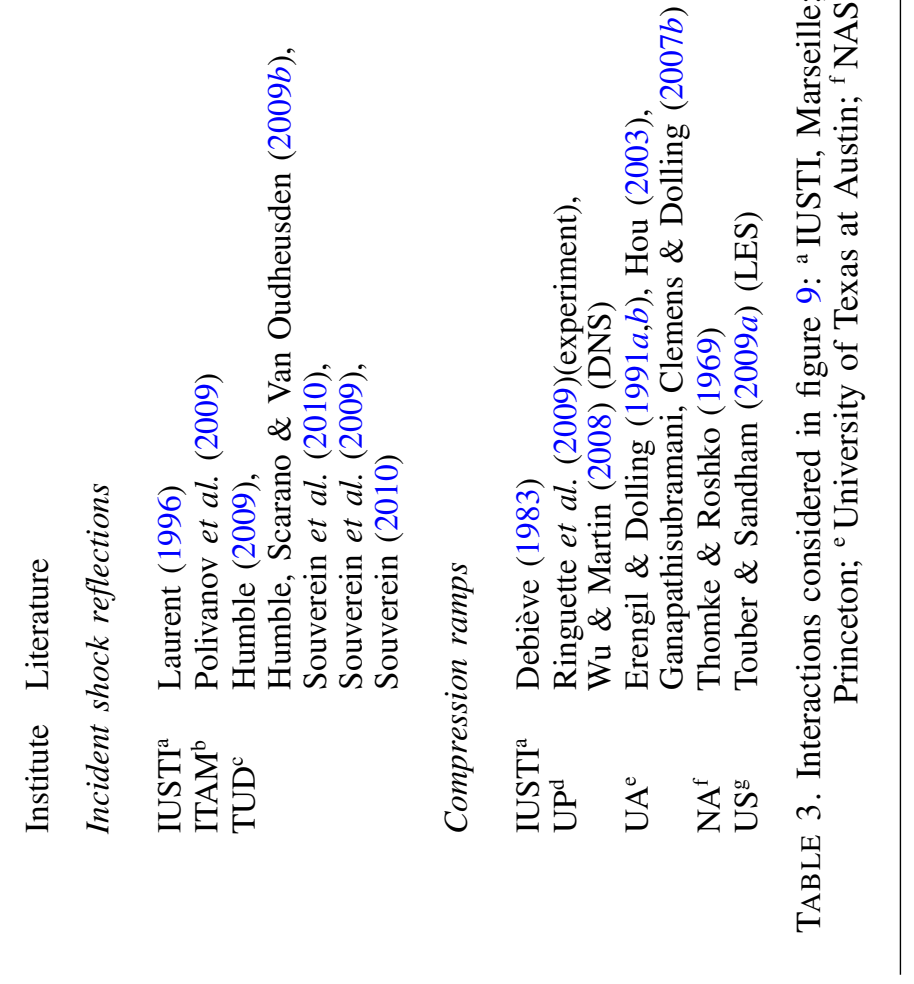
in the figures. Nevertheless, as shown in figure 9, they follow the general trend for any separation state or Mach number considered, confirming that the data are consistent with the proposed scaling. Similarly, the separation states of the heated wall experiments from IUSTI are not documented. They are shown in the figure, with the state of separation of the adiabatic interaction considered at the same flow deviation. Despite the uncertainty of the effect of wall heating on the separation state, and of the applicability of the approximation given by the relations (2.20) and (2.21) for non-adiabatic wall conditions (which could not be verified at this stage), this set of experiments follows the same general trend.

A best fit of a power law (see the equation in the figure legend) is given in figure 9. It corresponds to an $R^{2}$ value of 0.9428 . Good collapse of the whole set of data is obtained. Only the data from Polivanov et al. (2009) differ from the compilation. Although no significant effects have been found in these experiments on the dimensionless frequency (or Strouhal number) for the unsteady properties, it can be observed from their Schlieren visualizations that the reflected shock is relatively thick: this can be interpreted as substantive three-dimensional effects as well as important shock dynamics. It is not known whether the mean shock trace or the most upstream shock position has been used to determine the interaction length. The latter case would be in accordance with the approximate magnitude of the overestimation. In addition, this interaction appears to be of the transitional type $\left(R e_{x} \approx 2-3 \times 10^{6}\right)$. This regime probably requires a specific Reynolds-number-dependent scaling for $\Delta P_{\text {sep }}$. The other data follow the same trend as the data used in figure 7 , notwithstanding the fact that the data set now covers the full range from high-Reynolds-number experimental data (for example the TUD, UA, or NA experiments) down to low-Reynolds-number numerical results (the US Mach 2.3 reflection interaction case and the UP Mach 3 compression corner case). Using the new scaling, all points follow the best fit curve to within an error that is at least an order of magnitude smaller than the initial one (see figure 2) and about the same order as the expected measurement uncertainty: see $§ 2.1$. Note that apart from measurement uncertainties, part of the scatter on the rescaled data will certainly also have physical causes, i.e. effects that are not taken into account by the model (for instance effects induced by differences in the experimental setups, the flow facilities, etc.).

Given the scaling proposed in figure 9, at least two options for controlling the interaction can be suggested. To suppress flow separation, the trend line indicates that one should either act on $S_{e}^{*}$, imposing $\Delta P \rightarrow 0$, or on $L / \delta_{i n}^{*}$, imposing $\delta_{i n}^{*} \rightarrow 0$. The first option $(\Delta P \rightarrow 0)$ actually modifies the physical separation state of the flow. In the case of the second option, keeping the value of $S_{e}^{*}$ fixed (in other words, $\varphi=$ const. and $M_{e}=$ const.) means that the separation state is inherently prescribed, while $L / \delta_{i n}^{*}$ is a constant determined by the trend line. The second option hence implies that to reattach the flow, one should make $L$ vanish, which is achieved when $\delta_{i n}^{*} \rightarrow 0$, in other words when $R_{\delta^{*}} \rightarrow 0$. For upstream control, this appears to be the only option when the interaction geometry and free-stream conditions are fixed. We reiterate that this approach does not alter the flow separation state, but that it diminishes the separation bubble size by a reduction in the interaction size. Of course, these observations are subject to the assumptions used in the derivation of the scaling. For example, things might look differently locally if the flow becomes subject to significant spanwise modulations (three-dimensional effects). Nevertheless, in a global (spanwise averaged) sense they might, however, retain a certain validity in these cases too.

Finally, some comments can be derived concerning the three-dimensional effects in the SWBLI experiments. It is well known that wind tunnel experiments can 
develop significant three-dimensional organization in such flows, due to the lateral interactions developing on the sidewalls. In cases of separated interactions, large spanwise structures can develop in the separated region, even though the mean head shock remains qualitatively two-dimensional (see Dupont et al. 2005; Garnier 2009). In general, the reflection geometry is considered as more sensitive to such lateral effects than the compression corner configurations. Nevertheless, figure 9 does not highlight any discrepancies between these two families of SWBLIs, even for massive separated interactions, except perhaps for the Polivanov data (see previous comments). Therefore, we suggest that even though the present results could be subject to threedimensional effects, in a global (spanwise averaged) sense they might, however, retain a certain validity

\section{Discussion}

\subsection{Mach-Reynolds dependence}

The separation state criterion $S_{e}^{*}$ depends only slightly on the Reynolds number of the upstream boundary layer via the coefficient $k$ (see relations (2.21) and (2.24)), a dependence that seems to vanish in the limit of high Reynolds numbers $\left(R e_{\theta}>10^{4}\right)$. In contrast, there is a direct dependence on the Mach number and the imposed flow deflection angle (see (2.24)). It enables graphical determination of the Mach-deflection-angle combination, leading to a particular tendency of the flow to separate. An average value of $k$ of 2.8 has been used in this case. As can be observed from figure 10(b), for small Mach numbers, the incident shock deflection angle can be multiplied by two to obtain the equivalent ramp angle that yields the same value of $S_{e}^{*}$. From the figure it can be read that, for example, the same flow separation state should be expected for the Mach $=5, \varphi=28^{\circ}$ compression ramp as for the Mach $=3$, $\varphi=24^{\circ}$ ramp.

Observe that due to the Mach-number effect, the flow over a $\varphi=20^{\circ}$ ramp at Mach $=2$ should be more separated than a ramp at Mach $=3$ for the same deflection angle. This is clearly illustrated by figure 10(a), confirming the well-known property that an increase in Mach number tends to postpone the onset of flow separation at constant deflection angle (see also Ginoux 1973). Note that the current results equally indicate that an increase in $M_{e}$ leads to a reduction of the interaction length at constant $\varphi$ ( $S_{e}^{*}$ decreases and $G_{3}\left(M_{e}, \varphi\right)$ increases; figure 9 and (2.15) now show that $L^{*}$ must decrease and consequently also $L$ for constant $\delta_{i n}^{*}$ ). Further, note that for a Mach $=3$ interaction, the flow will always be separated for ramp angles larger than $\varphi=16-18^{\circ}$ (incident shock deflections of $\varphi=8-9^{\circ}$ ). Alternatively, a ramp of approximately $\varphi=6^{\circ}$ (an incident shock deflection of $\varphi=3^{\circ}$ ) will be attached for any Mach number that does not involve a normal shock solution.

\subsection{A new spatial scaling for turbulent fields in SWBLIs}

The common choice for the longitudinal length scale is the interaction length $L$. For the scaling of the wall-normal length scale, one might intuitively choose a characteristic length related to the upstream boundary layer (for example $\delta_{0}$ or $\delta^{*}$ ). However, such quantities can by definition not take into account the variations in interaction properties due to different imposed flow deflection angles. The question of the scaling of the wall-normal length scale will be addressed below, using data obtained in two supersonic facilities, namely the TST-27 wind tunnel at the Delft University of Technology and the S8 wind tunnel at the Institut Universitaire des Systèmes Thermiques Industriels (IUSTI), to verify the results. 

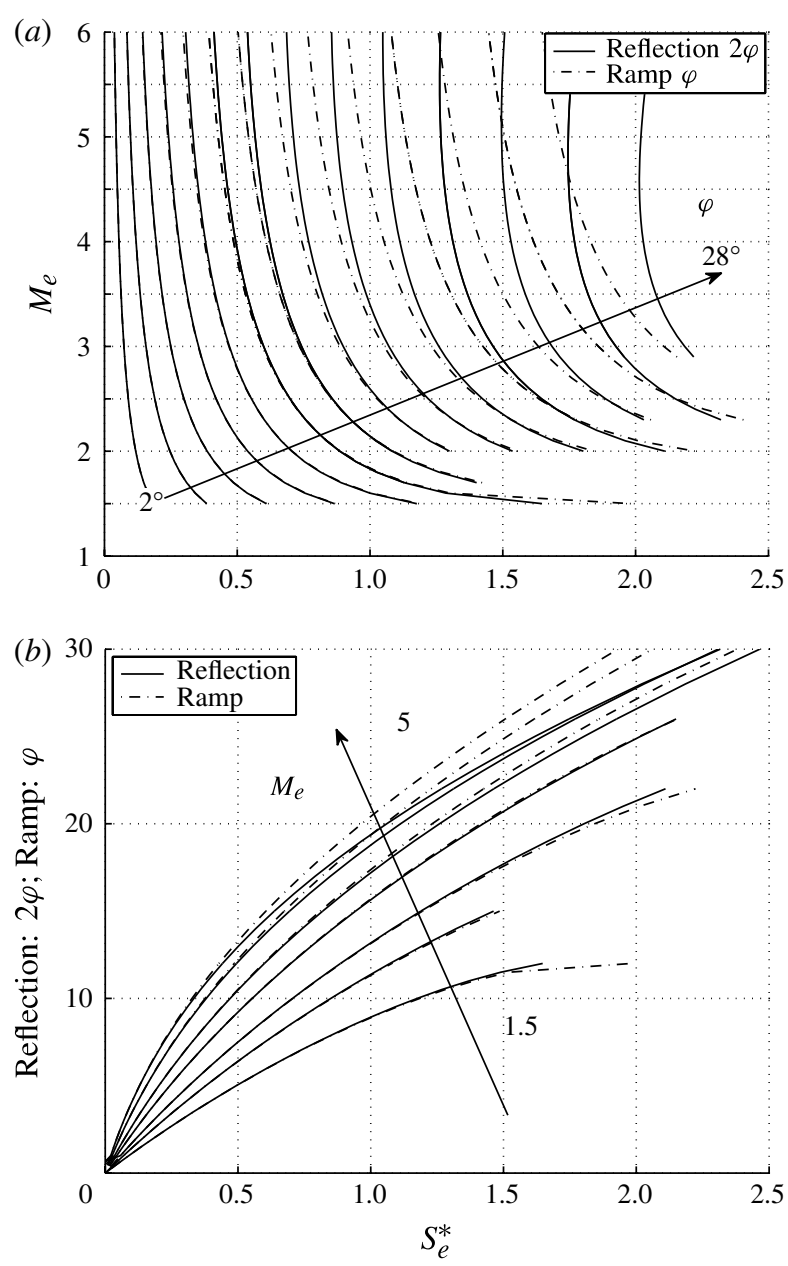

FIGURE 10. Dependence of $S_{e}^{*}$ on the Mach number and the flow deflection: $(a)$ Mach dependence $M_{e}\left(\varphi\right.$-discretization $\left.[2: 2: 28]^{\circ}\right) ;(b)$ flow deflection dependence $\varphi^{\circ}\left(M_{e^{-}}\right.$ discretization [ $\left[\begin{array}{llllll}1.5 & 1.75 & 2.0 & 2.5 & 3 & 4\end{array}\right]$ ). The figures include the theoretical dependence of $S_{e}^{*}$ on $M_{e}$ and $\varphi$ for both the incident reflection and the compression ramp.

The most important parameter differentiating the two facilities is the momentumthickness-based Reynolds number, which is a factor 10 higher for the TST-27 compared to the S8. The current investigation has taken advantage of the combined use of both facilities to obtain measurements of the same flow phenomenon over a range of measurement conditions (Reynolds number, Mach number, shock strength, interaction control), using different measurement systems, enabling a thorough comparison and validation of the observations. The flow cases under consideration have already been extensively documented (Dupont et al. 2005; Dussauge et al. 2006; Dupont et al. 2006, 2008; Souverein et al. 2009, 2010; Souverein \& Debiève 2010). The incoming boundary layers are turbulent and fully developed. The main aerodynamic parameters are listed in table 4 , where the boundary layer thickness $\delta_{0}$ was based on $99 \%$ of the external velocity $U_{e}, \delta^{*}$ is the displacement thickness, $\theta$ is the momentum thickness and $H$ the shape factor. 


$\begin{array}{lccccccc} & M_{e} & \varphi & \delta_{0}(\mathrm{~mm}) & \delta^{*}(\mathrm{~mm}) & \theta(\mathrm{mm}) & H & \operatorname{Re}_{\theta} \\ \text { TST-27 } & 1.69 & 6.0 & 17.2 & 3.3 & 1.4 & 2.4 & 50000 \\ \text { S8 } & 2.28 & 5.5 ; 8.0 ; 9.5 & 10.2 & 3.0 & 0.9 & 3.5 & 5000\end{array}$

TABLE 4. Measurement conditions.

The full field quantitative velocity data used in this investigation, including the mean and turbulent velocity fields, were obtained by means of PIV. The data acquisition and post-processing were done using the systems and software available in each laboratory.

(i) At TU Delft, a LaVision DaVis 7.2 acquisition system was used with a PTU 9 timing unit. Data processing was done with in-house developed WIDIM software employing an iterative multigrid deforming window correlation scheme (see Scarano \& Riethmuller 1999).

(ii) At IUSTI, a Dantec Dynamics system was used for the data acquisition, timing, and data processing.

In both cases, a dimensionless spatial resolution of $y / \delta \approx 2-2.9 \times 10^{-2}$ was used to define the PIV interrogation window size. The seeding systems were also different: Di*2-ethylhexyl sebacate (DEHS) droplets at TU Delft and incense smoke at IUSTI. See previous references for a detailed description of the experiments. In both facilities, the inflow conditions comprise a canonical zero pressure gradient supersonic $(M<5)$ turbulent boundary layer.

As detailed in table 4, four cases have been considered:

(i) two incipient cases in the two facilities, with Mach numbers of $M_{e}=1.7$ and 2.3 respectively;

(ii) two low-Reynolds-number separated interactions for $M_{e}=2.3$.

The incipient cases were adjusted to present similar probability of reverse flow, with a $50 \%$ probability near the wall (see Souverein et al. 2010).

The turbulent velocity fields obtained within the interaction regions were shown in Souverein et al. (2010). In all four cases, the turbulent fields exhibit a resemblance: a highly turbulent region develops downstream of the foot of the reflected shock, related to the formation of large coherent scales in the mixing layer which develops downstream of this point (Dupont et al. 2006, 2008; Souverein et al. 2009). Nevertheless, the extent of the development of this region was found to depend on the state of the interactions (incipient or separated): see figure 6 in Souverein et al. (2010).

The question of the physically correct scaling of the wall-normal length scale will now be readdressed. This will be done using the scaling analysis of $\S 2.2$. Indeed, it has been shown that only one length scale ( $\delta^{*}$, say) cannot suffice to describe the spatial extent of the interaction. Therefore, the non-dimensional length scale $L^{*}$ defined by (2.15) will be introduced. Using this equation one can scale the wall-normal coordinate by $L$ as follows:

$$
Y^{*}=\frac{y}{L G_{3}\left(M_{e}, \varphi\right)}=\frac{y}{\delta_{i n}^{*} L^{*}}
$$

where $G_{3}\left(M_{e}, \varphi\right)$ is an analytical function (see (2.16)).

Figure 11 shows the result obtained from scaling the wall-normal coordinate using (3.1). The extent of the vertical axis is the same for the four cases. The unit value for 
(a)
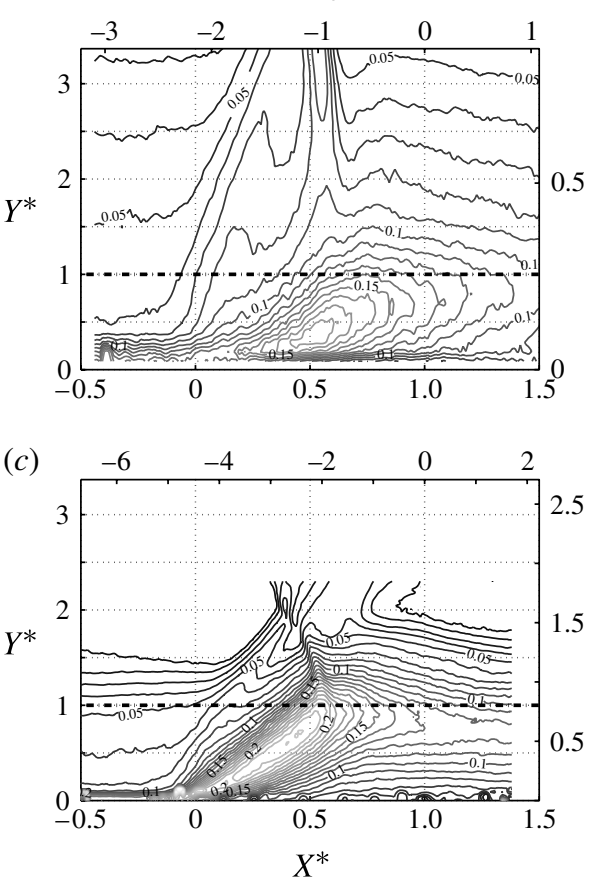

(b)

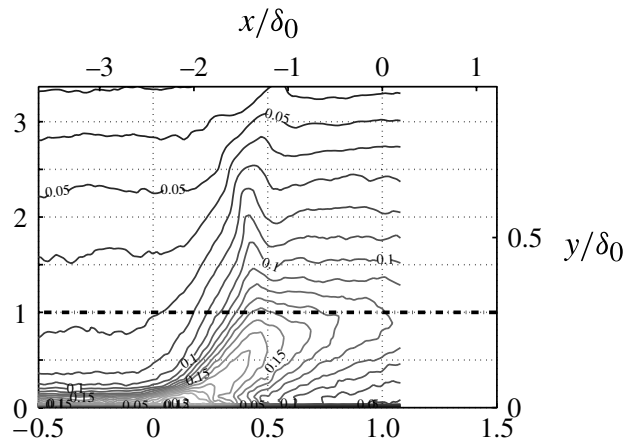

$(d)$

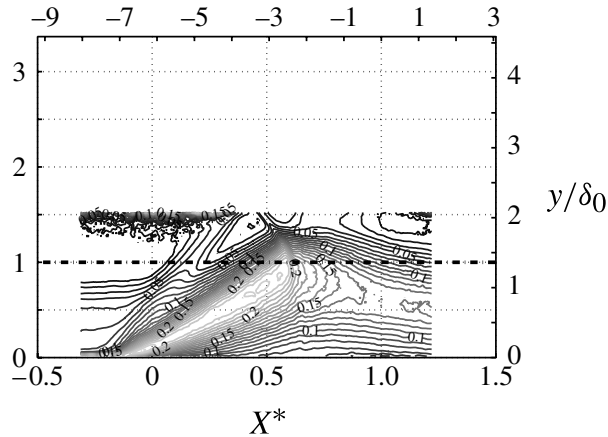

FIGURE 11. $U$-component fluctuations (root mean square) normalized by the free-stream velocity using the $L^{*}$ scaling option: $(a)$ high-Reynolds-number incipient case, $6.0^{\circ} ;(b)$ low-Reynolds-number incipient case, $5.5^{\circ}$; ( $c$ low-Reynolds-number separated case, $8.0^{\circ} ;(d$ low-Reynolds-number separated case, $9.5^{\circ}$. The fluctuation values are normalized by the freestream velocity. The black horizontal dashed line indicates the unit value for the $y$-axis. The horizontal and vertical scales on the top and right axes represent the coordinates normalized by $\delta_{0}$ for reference.

the $y$-axis, indicated by the black dashed line, corresponds to the approximate extent of the highly turbulent region developing downstream of the head shock. In all cases, the interactions are included in a $1 \times 1$ box, which shows that the scaling defined by (2.15) is relevant for the comparison of turbulent fields of different interactions.

\section{Conclusions}

A scaling analysis was made, aimed at reconciling the observed discrepancies between interactions documented in the literature (e.g. geometry, Reynolds-number effects, Mach-number effects, and shock intensity). As part of this analysis, a separation criterion has been formulated that depends only on the free-stream Mach number and the flow deflection angle. It successfully classifies the separation states for a large scope of documented interactions (compression ramp and incident-reflecting shock) over a large Reynolds-number and Mach-number range:

$$
\begin{aligned}
& S_{e}^{*}=k \frac{\Delta P}{q_{e}}=k g_{1}\left(M_{e}, \varphi\right), \\
& S_{e}^{*}<1 \quad \text { attached flow, } \\
& S_{e}^{*} \geqslant 1 \quad \text { separated flow, }
\end{aligned}
$$


where $k$ is a constant which depends slightly on the Reynolds number (see (2.21)). In addition, a scaling approach has been derived for the interaction length based on the mass balance:

$$
L^{*}=\frac{L}{\delta_{i n}^{*}} G_{3}\left(M_{e}, \varphi\right),
$$

with

$$
G_{3}\left(M_{e}, \varphi\right)=\frac{\sin (\beta) \sin (\varphi)}{\sin (\beta-\varphi)} .
$$

The separation criterion $S_{e}^{*}$ in combination with the normalized $L^{*}$ represents a single trend line onto which all data for a large scope of documented interactions over a large Reynolds and Mach range fall together with a moderate scatter of approximately $\pm 15 \%$, which is of the same order as the measurement uncertainty. This curve is valid both for compression ramp interactions and for incident-reflecting shock interactions. A similar formulation has been obtained from the momentum balance. The two results are equivalent.

A scaling for the wall-normal coordinate has been defined based on this scaling approach for the interaction length: $Y^{*}=y / L G_{3}\left(M_{e}, \varphi\right)$. It produces a large resemblance in the geometric organization of the turbulent flow fields within the interactions for the considered flow cases, independent of the Mach number and Reynolds number and of the separation state.

With regard to the Reynolds-number and Mach-number effects, it can be concluded that for turbulent boundary layers, the onset of flow separation is nearly Reynoldsnumber-independent. It seems to be governed principally by the Mach number and the imposed flow deflection angle: an increase in Mach number tends to reduce the deflection angle for the onset of flow separation. The Reynolds-number effects appear implicitly through the scaling of the interaction length by the displacement thickness of the incoming boundary layer. However, the interaction length is also governed by the Mach number via a correction factor that also involves the imposed flow deflection angle.

\section{Acknowledgements}

This research was a joint effort between TU Delft and IUSTI, and it was performed during the research period of L.J.S. at these institutions. Part of this work was carried out with support from CNES/ONERA Aérodynamique des Tuyères et Arrière-Corps (ATAC), and a grant of the European STREP UFAST (contract AST4-CT-2005012226). Their support is gratefully acknowledged.

\section{Appendix}

In analogy to the mass conservation approach in $\S 2.2$, the same analysis has been applied to the momentum conservation. This is done for both the incident shock reflection and the compression ramp case. Finally, a common formulation is derived. For completeness, note that the length scale for the momentum deficit is obtained by integrating the momentum flux within the boundary-layer profile (with $H$ the shape factor) as follows:

$$
\begin{aligned}
\frac{1}{\rho_{e} U_{e}^{2}} \int_{0}^{\infty}\left(\rho_{e} U_{e}^{2}-\rho U^{2}\right) \mathrm{d} y & =\int_{0}^{\infty} \frac{\rho U}{\rho_{e} U_{e}}\left(1-\frac{U}{U_{e}}\right) \mathrm{d} y+\int_{0}^{\infty}\left(1-\frac{\rho U}{\rho_{e} U_{e}}\right) \mathrm{d} y \\
& =\theta+\delta^{*}=\theta(1+H) .
\end{aligned}
$$




\section{Incident shock reflection}

First, the incident shock reflection case is analysed. Evaluating the $X$-momentum equation over the control volume shown in figure $5(a)$ the following equality is obtained for the inviscid flow case:

$$
\rho_{1} U_{1}^{2} H_{c v}+\rho_{2} U_{2} V_{2} L_{c v}-\rho_{3} U_{3}^{2} H_{c v}=\left(p_{3}-p_{1}\right) H_{c v} .
$$

At the same time, the following equality is obtained for the case with interaction:

$$
\begin{aligned}
& \rho_{1} U_{1}^{2}\left(H_{c v}-\theta_{1}\left(1+H_{1}\right)\right)+\rho_{2} U_{2} V_{2}\left(L_{c v}-L\right)-\rho_{3} U_{3}^{2}\left(H_{c v}-\theta_{3}\left(1+H_{3}\right)\right) \\
& \quad=\left(p_{3}-p_{1}\right) H_{c v} .
\end{aligned}
$$

Eliminating the pressure term, the following equation is obtained for the interaction length for the shock reflection case:

$$
L=\frac{\rho_{3} U_{3}^{2} \theta_{3}\left(1+H_{3}\right)-\rho_{1} U_{1}^{2} \theta_{1}\left(1+H_{1}\right) .}{\rho_{2} U_{2} V_{2}} .
$$

In analogy to the result from the mass conservation approach, the interaction length obtained from the momentum conservation approach is completely determined by the upstream and downstream boundary-layer momentum thicknesses and shape factors, the densities and velocities being specified by oblique shock-wave theory. The equation is independent of the height and length of the control volume as long as the interaction is included.

\section{Compression ramp}

Next, the compression ramp case is analysed. Evaluating the $X$-momentum equation over the control volume shown in figure $5(b)$, the following equality is obtained for the inviscid flow case:

$$
\rho_{1} U_{1}^{2} H_{c v_{1}}-\rho_{2} U_{2}^{2} H_{c v_{2}} \cos (\varphi)=\left(p_{2}-p_{1}\right) H_{c v_{1}} .
$$

At the same time, the following equality is obtained for the case with interaction:

$$
\begin{aligned}
& \rho_{1} U_{1}^{2}\left(H_{c v}-\theta_{1}\left(1+H_{1}\right)\right)-\rho_{2} U_{2}^{2} L \sin (\varphi) \cos (\varphi)-\rho_{2} U_{2}^{2}\left(H_{c v_{2}}-\theta_{2}\left(1+H_{2}\right)\right) \cos (\varphi) \\
& \quad=\left(p_{2}-p_{1}\right) H_{c v_{1}} .
\end{aligned}
$$

Eliminating the pressure term, the following equation is obtained for the interaction length for the compression ramp case:

$$
L=\frac{\rho_{2} U_{2}^{2} \theta_{2}\left(1+H_{2}\right) \cos (\varphi)-\rho_{1} U_{1}^{2} \theta_{1}\left(1+H_{1}\right)}{\rho_{2} U_{2}^{2} \sin (\varphi) \cos (\varphi)} .
$$

Again, the interaction length obtained from the momentum conservation approach is completely determined by the upstream and downstream boundary-layer momentum thicknesses and shape factors, the densities and velocities being specified by oblique shock-wave theory. The equation is independent of the height and length of the control volume as long as the interaction is included.

\section{Common formulation}

In analogy to the mass conservation approach, we attempt to find a common formulation for (A 4) and (A 7). To this end, the denominator of each equation is reformulated in terms of the upstream conditions by means of the oblique shock-wave 
relations. Using the fact that the velocity tangent to the shock is conserved, the following equality is obtained:

$$
U_{1} \cos (\beta)=U_{2} \frac{\cos (\beta-\varphi)}{\cos (\varphi)} .
$$

Similarly, considering mass conservation across the shock for the compression ramp case gives

$$
U_{1} \cos (\beta)=U_{2} \cos (\beta-\varphi) \text {. }
$$

Using (2.10) and (2.11), rewriting each of the equalities above and substituting all in their respective relations ((A 4) for the incident shock interaction and (A 7) for the compression corner) gives an equation of the same form, which can be written as follows, using the subscripts in and out for the inflow and outflow conditions respectively and defining the momentum deficit as $I^{*}=\rho U^{2} \theta(1+H)$ :

$$
\frac{L}{\theta_{\text {in }}\left(1+H_{\text {in }}\right)}=g_{3}\left(M_{e}, \varphi\right) g_{4}\left(M_{e}, \varphi\right)\left(\frac{I_{\text {out }}^{*} C(\varphi)}{I_{\text {in }}^{*}}-1\right),
$$

where

$$
\begin{aligned}
\text { shock reflection } C(\varphi) & =1 \\
\text { compression } \operatorname{ramp} C(\varphi) & =\cos (\varphi),
\end{aligned}
$$

and with $g_{3}\left(M_{e}, \varphi\right)$ a ratio of sine functions and $g_{4}\left(M_{e}, \varphi\right)$ a ratio of cosine functions,

$$
g_{3}\left(M_{e}, \varphi\right)=\frac{\sin (\beta-\varphi)}{\sin (\beta) \sin (\varphi)}, \quad g_{4}\left(M_{e}, \varphi\right)=\frac{\cos (\beta-\varphi)}{\cos (\beta) \cos (\varphi)} .
$$

The factor $C(\varphi)$ appears in the compression ramp equation due to the definition of $U_{\text {out }}$ : the $X$-momentum is considered, while the outgoing velocity is aligned with the ramp. The equation gives an alternative algebraic relation for the interaction length as a function of $M_{e}, \varphi$, the incoming boundary-layer fullness, and the momentum deficit ratio between the incoming boundary layer and the outgoing boundary layer. In analogy to the mass conservation result, it can be concluded that the interaction length is also a direct consequence of the momentum deficit ratio between the incoming and outgoing boundary layer. The proper scaling for the vertical axis in terms of the momentum deficit ratio, with $\hat{L}$ the non-dimensional interaction length and with $C(\varphi)$ defined by (A 10), would hence be

$$
\hat{L}=\frac{L}{\theta_{\text {in }}\left(1+H_{\text {in }}\right)} G_{3}\left(M_{e}, \varphi\right) G_{4}\left(M_{e}, \varphi\right)=\frac{I_{\text {out }}^{*}}{I_{\text {in }}^{*}} C(\varphi)-1,
$$

where

$$
\begin{aligned}
& G_{3}\left(M_{e}, \varphi\right)=g_{3}\left(M_{e}, \varphi\right)^{-1}=\frac{\sin (\beta) \sin (\varphi)}{\sin (\beta-\varphi)}, \\
& G_{4}\left(M_{e}, \varphi\right)=g_{4}\left(M_{e}, \varphi\right)^{-1}=\frac{\cos (\beta) \cos (\varphi)}{\cos (\beta-\varphi)} .
\end{aligned}
$$

\section{REFERENCES}

Anderson, J. D. JR 1991 Fundamentals of Aerodynamics, 2nd edn. McGraw-Hill.

ARDONCEAU, P. L. 1984 The structure of turbulence in a supersonic shock-wave/boundary-layer interaction. AIAA J. 22 (9), 1254-1262. 
AtKin, C. J. \& SQUiRe, L. C. 1992 A study of the interaction of a normal shock wave with a turbulent boundary layer at Mach numbers between 1.30 and 1.55. Eur. J. Mech. 11 (1), 93-118.

Benkemoun, L. \& Salaun, M. 1988 Développement d'une couche limite turbulente supersonique sur une paroi chauffée: propriétés du champ turbulent et exploitation théorique. Tech. Rep, ONERA Rept. n74/7078.

Beresh, S. J., Clemens, N. T. \& Dolling, S. D. 2002 Relationship between upstream turbulent boundary layer velocity fluctuations and separation shock unsteadiness. AIAA J. 40 (12), 2412-2422.

Bourgoing, A. \& Reijasse, P. H. 2005 Experimental analysis of unsteady separated flows in a supersonic planar nozzle. Shock Waves 14 (4), 251-258.

BRUCE, P. J. K. \& BABINSKY, H. 2008 Unsteady shock wave dynamics. J. Fluid Mech. 603, 463-473.

Brusniak, L. \& Dolling, D. S. 1994 Physics of unsteady blunt-fin-induced shock wave/turbulent boundary layer interactions. J. Fluid Mech. 273, 375-409.

Bueno, P. C. 2006 The effects of upstream mass injection by vortex generator jets on shock-induced turbulent boundary layer separation. Doctoral thesis, The University of Texas at Austin.

Bur, R., Benay, R., Galli, A. \& Berthouze, P. 2008 Experimental and numerical study of forced shock-wave oscillations in a transonic channel. Aerosp. Sci. Technol. 10, 265-278.

Chapman, D. R., Kuehn, D. M. \& LARson, H. K. 1957 Investigation of separated flow in supersonic and subsonic streams with emphasis on the effect of transition. Tech. Rep, NACA, Technical Note 3869.

Cherry, N. J., Hillier, R. \& Latour, M. E. M. 1984 Unsteady measurements in a separated and reattaching flow. J. Fluid Mech. 144, 13-46.

Clemens, N. T. \& Narayanaswamy, V. 2009 Shock/turbulent boundary layer interactions: review of recent work on sources of unsteadiness. In 39th Fluid Dynamics Conference and Exhibit, San Antonio, Texas, AIAA Paper 2009-3710.

DebiÈve, J. F. 1983 Etude d'une interaction turbulence-onde de choc. Thèse d'etat, Université d'Aix-Marseille.

DÉlery, J. M. \& Dussauge, J. P. 2009 Some physical aspects of shock wave/boundary layer interactions. Shock Waves 19 (6), 453-468.

Délery, J. M. \& MARVin, J. G. 1986 Shock wave-boundary layer interactions. Tech. Rep, AGARDograph no. 280.

Dolling, D. S. 2001 Fifty years of shock-wave/boundary-layer interaction research: what next? AIAA J. 39 (8), 1517-1531.

Dolling, D. S. \& BRUSNiAK, L. 1989 Separation shock motion in fin, cylinder, and compression ramp-induced turbulent interactions. AIAA J. 27 (6), 734-742.

Dolling, D. S. \& MURPhY, M. T. 1983 Unsteadiness of the separation shock wave structure in a supersonic compression ramp flow field. AIAA J. 21 (12), 1628-1634.

Dolling, D. S. \& OR, C. T. 1985 Unsteadiness of the shock wave structure in attached and separated compression ramp flows. Exp. Fluids 3, 24-32.

Dupont, P., Haddad, C., Ardissone, J. P. \& Debiève, J. F. 2005 Space and time organisation of a shock wave/turbulent boundary layer interaction. Aerosp. Sci. Technol. 9 (7), 561-572.

Dupont, P., Haddad, C. \& Debiève, J. F. 2006 Space and time organization in a shock-induced boundary layer. J. Fluid Mech. 559, 255-277.

Dupont, P., Piponniau, S., Sidorenko, A. \& Debiève, J. F. 2008 Investigation of an oblique shock reflection with separation by PIV measurements. AIAA J. 46 (6), 1365-1370.

Dussauge, J. P., Dupont, P. \& Debiève, J. F. 2006 Unsteadiness in shock wave boundary layer interactions with separation. Aerosp. Sci. Technol. 10, 85-91.

Erengil, M. E. \& Dolling, D. S. 1991a Correlation of separation shock motion with pressure fluctuations in the incoming boundary layer. AIAA J. 29 (11), 1868-1877.

ERengil, M. E. \& Dolling, D. S. $1991 b$ Unsteady wave structure near separation in a Mach 5 compression ramp interaction. AIAA J. 29 (5), 728-735. 
Frey, M. \& Hagemann, G. 1998 Status of flow separation prediction in rocket nozzles. In 34th Joint Propulsion Conference and Exhibit. AIAA Paper 98-3619.

Frey, M. \& Hagemann, G. 2000 Restricted shock separation in rocket nozzles. J. Propul. Power 16 (3), 478-484.

Ganapathisubramani, B., Clemens, N. T. \& Dolling, D. S. $2007 a$ Effects of upstream boundary layer on the unsteadiness of shock-induced separation. J. Fluid Mech. 585, 369-394.

Ganapathisubramani, B., Clemens, N. T. \& Dolling, D. S. $2007 b$ Effects of upstream coherent structures on low-frequency motion of shock-induced turbulent separation. In 45th AIAA Aerospace Sciences Meeting and Exhibit, Reno, Nevada, AIAA paper 2007-1141.

GARNIER, E. 2009 Stimulated detached eddy simulation of three-dimensional shock/boundary layer interaction. Shock Waves 19 (6), 479-486.

Ginoux, J. J. 1973 Interaction entre ondes de choc et couches limites. In Chocs et Ondes de Choc, vol. 2 (ed. A. L. Jaumotte). Masson \& Cie.

GREen, J. E. 1970 Reflexion of an oblique shock wave by a turbulent boundary layer. J. Fluid Mech. 40, 81-95.

Hou, Y. X. 2003 Particle image velocimetry study of shock-induced turbulent boundary layer separation. Doctoral thesis, The University of Texas at Austin.

Humble, R. A. 2009 Unsteady flow organization of a shock wave/boundary layer interaction. Doctoral thesis, Delft University of Technology.

Humble, R. A., Elsinga, G. E., Scarano, F. \& Van Oudheusden, B. W. $2009 a$ Three-dimensional instantaneous structure of a shock wave/turbulent boundary layer interaction. J. Fluid Mech. 622, 33-62.

Humble, R. A., Scarano, F. \& Van Oudheusden, B. W. $2009 b$ Unsteady aspects of an incident shock wave/turbulent boundary layer interaction. J. Fluid Mech. 635, 47-74.

KIYA, M. \& SASAKI, K. 1983 Structure of a turbulent separation bubble. J. Fluid Mech. 137, 83-113.

Kuntz, D. W., Amatucci, V. A. \& Addy, A. L. 1987 Turbulent boundary-layer properties downstream of the shock-wave/boundary-layer interaction. AIAA J. 25 (5), 668-675.

LAURENT, H. 1996 Turbulence d'une interaction onde de choc-couche limite sur paroi plane adiabatique ou chauffée. Thèse de 3ème cycle, Université d'Aix-Marseille II.

Piponniau, S. 2009 Instationnarités dans les décollements compressibles: cas des couches limites soumises à ondes de choc. Thèse de doctorat, Université de Provence.

Piponniau, S., Dussauge, J. P., Debiève, J. F. \& Dupont, P. 2009 A simple model for low-frequency unsteadiness in shock-induced separation. J. Fluid Mech. 629, 87-108.

Pirozzoli, S., Beer, A., Bernardini, M. \& Grasso, F. 2009 Computational analysis of impinging shock-wave boundary layer interaction under conditions of incipient separation. Shock Waves 19 (6), 487-497.

Pirozzoli, S. \& Grasso, F. 2006 Direct numerical simulation of impinging shock wave/turbulent boundary layer interaction at $M=2.25$. Phys. Fluids 18, 065113.

PLOTKIN, K. J. 1975 Shock wave oscillation driven by turbulent boundary layer fluctuations. AIAA J. 13 (8), 1036-1040.

Polivanov, P., Sidorenko, A. \& Maslov, A. 2009 Report on the measurement of shock reflection by Mach=2.0: final report. UFAST Deliverable 3.3.6, Institute of Theoretical and Applied Mechanics.

Ringuette, M. J., Wu, M. \& Martin, M. P. 2008 Low Reynolds number effects in a Mach 3 shock/turbulent-boundary-layer interaction. AIAA J. 46 (7), 1884-1887.

Ringuette, M. J., Bookey, P., Wyckham, C. \& Smits, A. J. 2009 Experimental study of a Mach 3 compression ramp interaction at $R e_{\theta}=2400$. AIAA J. 47 (2), 373-385.

SCARANO, F. \& Riethmuller, M. L. 1999 Iterative multigrid approach in PIV image processing with discrete window offset. Exp. Fluids 26, 513-523.

SCHMUCKER, R. 1973 Strömungsvortgänge beim Betrieb überexpandierter Düsen chemischer Raketentriebwerke (Flow processes in overexpanded nozzles of chemical rocket engines). Tech. Rep., Report TUM-LRT-TB-7,-10,-14, Technical University Munich, Munich. 
Selig, M. S., Andreopoulos, J., Muck, K. C., Dussauge, J. P. \& Smits, A. J. 1989 Details of a shock-separated turbulent boundary layer at a compression corner. AIAA J. 27 (7), 862-869.

Settles, G. S., Bogdonoff, S. M. \& VAs, I. E. 1976 Incipient separation of a supersonic turbulent boundary layer at high Reynolds number. AIAA J. 14 (1), 50-56.

Settles, G. S., Fitzpatrick, T. J. \& Bogdonoff, S. M. 1979 Detailed study of attached and separated compression corner flow fields in high Reynolds number supersonic flow. AIAA $J$. 17, 579-585.

Smits, A. J. \& Dussauge, J. P. 2006 Turbulent Shear Layers in Supersonic Flow, 2nd edn. AIP.

SMits, A. J. \& MuCK, K. C. 1987 Experimental study of three shock wave/turbulent boundary layer interactions. J. Fluid Mech. 182, 291-314.

SouvereIN, L. J. 2010 On the scaling and unsteadiness of shock induced separation. Doctoral thesis, Delft University of Technology-Université de Provence.

Souverein, L. J. \& Debiève, J. F. 2010 Effects of air jet vortex generators on a shock wave boundary layer interaction. Exp. Fluids 49 (5), 1053-1064.

Souverein, L. J., Dupont, P., Debiève, J. F., Dussauge, J. P., Van Oudheusden, B. W. \& SCARANO, F. 2010 Effect of interaction strength on unsteadiness in turbulent shock-wave-induced separations. AIAA J. 48 (7), 1480-1493.

Souverein, L. J., Van Oudheusden, B. W., Scarano, F. \& Dupont, P. 2009 Application of a dual-plane particle image velocimetry (dual-PIV) technique for the unsteadiness characterization of a shock wave turbulent boundary layer interaction. Meas. Sci. Technol. 20 (7), 074003.

SPAID, F. W. \& FRISHETT, J. C. 1972 Incipient separation of a supersonic, turbulent boundary layer, including effect of heat transfer. AIAA J. 10 (7), 915-922.

Summerfield, M., Foster, C. \& Swan, W. 1954 Flow separation in overexpanded supersonic exhaust nozzles. Tech. Rep. 24, Jet Propulsion Laboratory.

Thomas, F. O., Putman, C. M. \& ChU, H. C. 1994 On the mechanism of unsteady shock oscillation in shock wave/turbulent boundary layer interaction. Exp. Fluids 18, 69-81.

Tномке, G. J. \& Roshко, A. 1969 Incipient separation of a turbulent boundary layer at high Reynolds number in two-dimensional supersonic flow over a compression corner. Tech. Rep. DAC-59819, NASA-Ames Research Center.

TOUBER, E. \& SANDHAM, N. D. 2008 Oblique shock impinging on a turbulent boundary layer: low-frequency mechanisms. In 38th AIAA Fluid Dynamics Conference, Seattle, Washington, doi:10.2514/6.2008-4170.

Touber, E. \& SAndham, N. D. $2009 a$ Comparison of three large-eddy simulations of shock-induced turbulent separation bubbles. Shock Waves 19 (6), 469-478.

Touber, E. \& SAndham, N. D. $2009 b$ Large-eddy simulation of low-frequency unsteadiness in a turbulent shock-induced separation bubble. Theor. Comput. Fluid Dyn. 23 (2), 79-107.

Touber, E. \& SANDhAM, N. D. 2011 Low-order stochastic modelling of low-frequency motions in reflected shock-wave/boundary-layer interactions. J. Fluid Mech. 671 (3), 417-465.

ÜNAlmis, Ö. H. \& Dolling, D. S. 1996 On the possible relationship between low frequency unsteadiness of shock-induced separated flow and Goertler vortices. In 27th AIAA Fluid Dynamics Conference, New Orleans, Louisiana, AIAA Paper 1996-2002.

VISWANATH, P. R. 1988 Shock-wave-turbulent-boundary-layer interaction and its control: a survey of recent developments. Sādhanā 12, 45-104.

WU, M. \& MARTin, M. P. 2008 Analysis of shock motion in shockwave and turbulent boundary layer interaction using direct numerical simulation data. J. Fluid Mech. 594, 71-83.

ZUKOSKI, E. 1967 Turbulent boundary-layer separation in front of a forward-facing step. AIAA J. 5 (10), 1746-1753. 